\title{
On Spectral Asymptotics for Domains with Fractal Boundaries
}

\author{
S. Molchanov, B. Vainberg \\ Dept. of Mathematics, Univ. of North Carolina at Charlotte, Charlotte, NC 28223, USA
}

Received: 24 May 1995/Accepted: 2 April 1996

\begin{abstract}
We discuss the spectral properties of the Laplacian for domains $\Omega$ with fractal boundaries. The main goal of the article is to find the second term of spectral asymptotics of the counting function $N(\lambda)$ or its integral transformations: $\Theta$-function, $\zeta$-function. For domains with smooth boundaries the order of the second term of $N(\lambda)$ (under "billiard condition") is one half of the dimension of the boundary. In the case of fractal boundaries the well-known Weyl-Berry hypothesis identifies it with one half of the Hausdorff dimension of $\partial \Omega$, and the modified Weyl-Berry conjecture with one half of the Minkowski dimension of $\partial \Omega$. We find the spectral asymptotics for three natural broad classes of fractal boundaries (cabbage type, bubble type and web type) and show that the Minkowski dimension gives the proper answer for cabbage type of boundaries (due to "one dimensional structure" of the cabbage type fractals), but the answers are principally different in the two other cases.
\end{abstract}

\section{Contents}

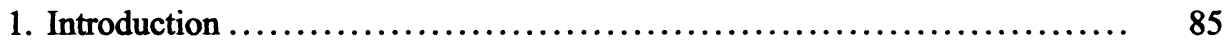

2. Cabbage type domains ......................................... 90

3. Bubble type domains $\ldots \ldots \ldots \ldots \ldots \ldots \ldots \ldots \ldots \ldots \ldots \ldots \ldots \ldots \ldots \ldots \ldots \ldots \ldots \ldots, 101$

4. Planar bubble fractals $\ldots \ldots \ldots \ldots \ldots \ldots \ldots \ldots \ldots \ldots \ldots \ldots \ldots \ldots \ldots \ldots \ldots \ldots \ldots \ldots, 112$

\section{Introduction}

The classical Weyl-Berry conjecture is related to the spectral counting function $N(\lambda)$ for the Laplacian in a bounded domain $\Omega \subset \Re^{d}, d \geqq 1$, with smooth boundary $\partial \Omega$. Let us consider the spectral problems for the Dirichlet Laplacian $-\Delta^{-}$:

$$
-\Delta \Psi=\lambda \Psi \quad \text { on } \Omega, \quad \Psi=0 \text { on } \partial \Omega
$$


and the Neumann Laplacian $-\Delta^{+}$:

$$
-\Delta \Psi=\lambda \Psi \quad \text { on } \Omega, \quad \frac{\partial \Psi}{\partial n}=0 \quad \text { on } \partial \Omega .
$$

Let

$$
N^{ \pm}(\lambda)=\#\left\{\lambda_{i}^{ \pm}<\lambda\right\}
$$

be a counting function for the eigenvalues $\lambda_{i}^{ \pm}$of the operators $-\Delta^{ \pm}$. Weyl's conjecture has the form

$$
N^{ \pm}(\lambda)=c_{0}(d)|\Omega| \lambda^{d / 2} \pm c_{1}(d)|\partial \Omega| \lambda^{(d-1) / 2}+o\left(\lambda^{(d-1) / 2}\right), \quad \lambda \rightarrow \infty,
$$

where $c_{0}, c_{1}$ are constants depending only on the dimension $d$ of the phase space, $|\Omega|$ is the volume of $\Omega,|\partial \Omega|$ is the area of the boundary surface.

The standard method of study of $N(\lambda)$ is based on its integral transformations. The simplest one is the following:

$$
\theta^{ \pm}(t):=\int_{-\infty}^{\infty} e^{-\lambda t} d N^{ \pm}(\lambda)=\int_{\Omega} p^{ \pm}(t, x, x) d x=\operatorname{Tr} e^{t \Delta^{ \pm}},
$$

where $p^{ \pm}(t, x, y)$ are the Green functions for the heat equation $\frac{\partial p}{\partial t}=\Delta p$ with Dirichlet or Neumann boundary condition.

Instead of $\theta^{ \pm}$-functions one can work with $\varsigma$-functions (or resolvents):

$$
\varsigma^{ \pm}(\lambda):=\sum_{i} \frac{1}{\left(\lambda+\lambda_{i}^{ \pm}\right)^{z}}=\int_{-\infty}^{\infty} \frac{d N^{ \pm}(\mu)}{(\lambda+\mu)^{z}}=\operatorname{Tr}\left(\lambda-\Delta^{ \pm}\right)^{-z}
$$

or with the Fourier transform of $d N^{ \pm}(\lambda)$ (which leads to the wave equation).

For domains with smooth boundaries the asymptotic expansions of the integral transformations are well known. Say

$$
\begin{gathered}
\theta^{ \pm}(t) \sim \sum_{k=0}^{\infty} a_{k}(\Omega) t^{(-d+k) / 2}, \quad t \rightarrow 0, \\
a_{0}(\Omega)=\frac{d}{2} \Gamma\left(\frac{d}{2}\right) c_{0}(d)|\Omega|, \quad a_{1}(\Omega)= \pm \frac{d-1}{2} \Gamma\left(\frac{d-1}{2}\right) c_{1}(d)|\partial \Omega|
\end{gathered}
$$

(Minakshasandaram expansion, see, for example $[\mathrm{McSi}, \mathrm{Ka}, \mathrm{Mo}])$. Here $\Gamma(s)$ is the Gamma function. A similar expansion is valid for $\varsigma^{ \pm}(\lambda), \lambda \rightarrow \infty$. The formal inversion of these expansions gives Weyl's expansions (1) for $N^{ \pm}(\lambda)$. However this inversion is valid only for the first term of $N^{ \pm}(\lambda)$ :

$$
N^{ \pm}(\lambda)=c_{0}(d)|\Omega| \lambda^{d / 2}(1 \pm o(1)), \quad \lambda \rightarrow \infty
$$

(Weyl's law). The remainder can be specified (Seeley [Se]):

$$
N^{ \pm}(\lambda)=c_{0}(d)|\Omega| \lambda^{d / 2}+O\left(\lambda^{\frac{d-1}{2}}\right), \quad \lambda \rightarrow \infty .
$$

As for the second term in (1) it's known that in the general smooth case formula (1) is valid under an additional "billiard condition" (Ivrii [Iv1, Iv2]). Let us mention that Ivrii's condition is generic, but it can be checked only for several simplest cases. 
If the boundary is very irregular then the question about billiard condition cannot be posed at all because the billiard trajectories are not determined.

If $\Omega$ is an open set, $|\Omega|<\infty$, and $\partial \Omega$ is irregular, then one can determine the Dirichlet and Neumann Laplacian in terms of the closure of the Dirichlet form

$$
D(f, g)=\int_{\Omega}(\nabla f, \nabla g) d x
$$

on the spaces $C_{0}^{\infty}(\Omega), C^{\infty}(\bar{\Omega})$ correspondingly. For the Dirichlet Laplacian the spectrum is discrete, and the leading term for $N^{-}(\lambda)$ (Weyl's law) has the same form (5), Melrose [Me].

The main goal of our paper is to find "the second term" of the spectral asymptotics in the case when the boundary of the domain is irregular (fractal). We'll discuss the generalized form of the Weyl's conjecture (1) (in most cases in a weaker form: not for $N(\lambda)$, but for it's integral transformations (2), (3)).

We shall work only with the Dirichlet Laplacian because for the Neumann Laplacian the spectrum, generally speaking, is not discrete and the counting function $N^{+}(\lambda)$ does not exist even when the domain has only one irregular point on $\partial \Omega$. The simplest example of such a domain is given by the union of open nonintersecting balls $B\left(x_{n} ; r_{n}\right)$ with radii $r_{n}$ and centers at $x_{n}$ (Fig. 1a). In this case $\lambda=0$ is the point of $S p_{\text {ess }}\left(-\Delta^{+}\right)$. B. Simon [Si] (see also [JaMoSi]) showed that the Neumann Laplacian in a bounded domain may have an absolutely continuous spectrum.

Let's return to the Dirichlet Laplacian. In the well-known paper [Be] M. Berry discussed the diffraction and scattering of waves by rough ("fractal") surfaces and formulated the following physical hypothesis (Weyl-Berry conjecture): if boundary $\partial \Omega$ has Hausdorff dimension $h=h(\partial \Omega)<d$ and corresponding Hausdorff measure $|\partial \Omega|_{h}$, then

$$
N^{-}(\lambda)=c_{0}(d)|\Omega| \lambda^{d / 2}-c_{1}(d, h)|\partial \Omega|_{h} \lambda^{h / 2}+o\left(\lambda^{h / 2}\right), \quad \lambda \rightarrow \infty .
$$

The spirit of this conjecture can be traced back to the classification of fractals by their Hausdorff dimensions which became very popular after Mandelbrot's book [Ma] on fractals in nature. However very soon Brassard and Carmona [BrCa] showed that Berry's hypothesis fails and constructed corresponding counterexamples. In fact it is possible to give a very simple example of such a type. One can consider the system of balls $B\left(x_{n} ; r_{n}\right)$ with $\sum r_{n}^{d}<\infty$ (Fig. 1b) and with Dirichlet boundary condition. The spectrum and $N^{-}(\lambda)$ don't depend on the location of the balls, but only on the set $\left\{r_{n}\right\}$. On the other hand different rearrangements of the balls in the space

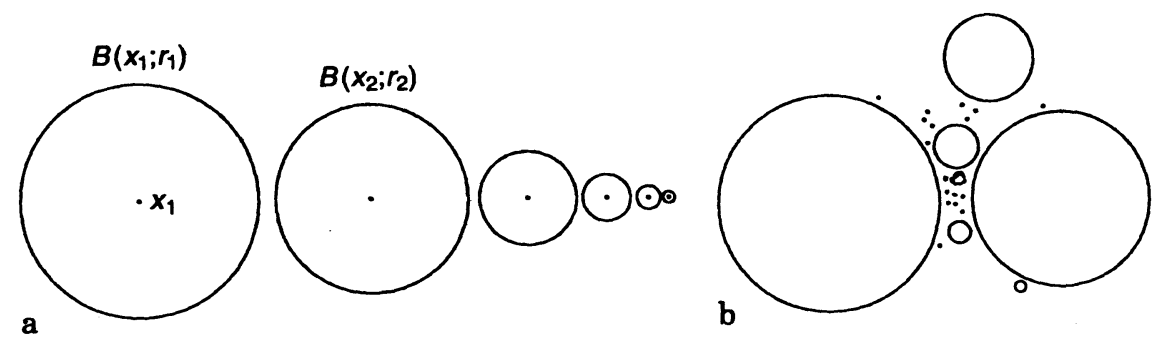

Fig. 1 
(for fixed $\left\{r_{n}\right\}$ !) can generate arbitrary Hausdorff dimension $h(\partial \Omega)$ in the interval $[d, d-1]$.

The physical reason why the Hausdorff dimension cannot be used for the description of the fractal boundary in spectral problems is trivial: the Hausdorff dimension describes the "content" of the boundary as a geometrical set of points, but it is not related to the description of the boundary layer of the domain.

Weyl-Berry conjecture with the Minkowski dimension $m=m(\partial \Omega)$ and the Minkowski content $|\partial \Omega|_{m}$ in formula (7) instead of the Hausdorff dimension and the Hausdorff measure is known as the modified Weyl-Berry conjecture (MWB conjecture). M. Lapidus in a long series of papers (see [La1, La2] and references there) proved a few essential results, supporting the MWB conjecture. In particular he proved that in the one-dimensional case the MWB hypothesis is valid [Lal]. In [La1] one can also find necessary and sufficient conditions for Minkowski measurability of the $\partial \Omega$ for an open set $\Omega \subset \Re$. Together with J. Fleckinger they showed $[\mathrm{LaF} 1, \mathrm{La} 2]$ that for any dimension $d$

$$
N^{-}(\lambda)=c_{0}(d)|\Omega| \lambda^{d / 2}+O\left(\lambda^{m / 2}\right), \quad \lambda \rightarrow \infty .
$$

Hua and Sleeman [HuSl] found effective constants $C_{ \pm}$such that the remainder in (8) can be estimated from above and below by $C_{ \pm} \lambda^{m / 2}$. The estimate from below is proved under an additional strong geometrical condition on $\Omega$ (existence of a suitable tessellation).

It turned out to be the case that the modified Weyl-Berry conjecture also fails. The simplest "argument" was mentioned in [BrCa, FlVa2]: one can remove a countable set of isolated points from the domain without changing $N(\lambda)$, but if these points are judiciously chosen, we can vary the Minkowski dimension and Minkowski content at will. However this argument requires only a slight specification of the conjecture: the only regular by Wiener part of the boundary has to be taken into account in the conjecture. This specification is very natural because boundary conditions cannot be posed at isolated points, and these points must be removed from the boundary set before evaluating the Minkowski dimension. The essential examples were studied by $\mathrm{J}$. Fleckinger and D. Vasiliev [FlVa, FlVa2] and later for a wider class of domains by M. Levitin and D. Vassiliev [LeVa]. They constructed selfsimilar fractals such that

$$
N^{-}(\lambda)=c_{0}(d)|\Omega| \lambda^{d / 2}-c_{1}(d, \lambda, \partial \Omega) \lambda^{m / 2}+o\left(\lambda^{m / 2}\right), \quad \lambda \rightarrow \infty,
$$

where the function $c_{1}$ is bounded and strictly positive, but oscillates as $\lambda \rightarrow \infty$ (see [FlVa]). M. Lapidus and C. Pomerance [LaPo] gave another example where formula (7) is valid but the coefficient $c_{1}$ cannot be expressed through the Minkowski content $|\partial \Omega|_{m}$ of the boundary. Even though these examples disprove the modified WeylBerry conjecture they support its main part because the order of the second term of $N^{-}(\lambda)$ in these examples is equal exactly to $m / 2$.

The main purpose of the present work is to single out broad classes of fractals (without assumptions on selfsimilarity or geometrical conditions which allow to separate variables) for which spectral asymptotics can be found. The second goal was to answer the following question: is the order of the second term of spectral asymptotics always related to the Minkowski dimension of the boundary? In particular, suppose that $N^{-}(\lambda)$ has two terms of asymptotics as $\lambda \rightarrow \infty$ :

$$
N^{-}(\lambda)=c_{0}(d)|\Omega| \lambda^{d / 2}-c_{1}(d, \partial \Omega) \lambda^{s / 2}+o\left(\lambda^{s / 2}\right), \quad \lambda \rightarrow \infty
$$


(we can call $s=s(\partial \Omega)$ as a spectral dimension of $\partial \Omega$ ). Is it true or not, that $s(\partial \Omega)=$ $m(\partial \Omega)$ ? Of course it is true for $d=1$ due to the Lapidus result. For dimensions $d>1$ formula (8) leads to the inequality $s \leqq m$. The answer for the last question is complicated and more often negative. First of all let us mention that the second (boundary) term of the spectral asymptotics may not exist or may have a more complicated form than in (9). But even if the asymptotic formula (9) is valid, then the spectral dimension $s$ in the majority of interesting cases from the physical point of view is not connected with the Minkowski dimension of $\partial \Omega$, and it has an absolutely different geometrical (physical) meaning.

Let us mention that the term "domain with a fractal boundary" may have different meanings. One can understand it as a domain whose boundary is a closed fractal set in the Mandelbrot [Ma] sense, i.e. the boundary of the domain is a connected Cantor type set with an additional hierarchical (selfsimilar) structure. The physical idea of fractals in nature is different (see M. Berry [Be]). These are usually objects like clouds, Earth lithosphere, Solar magnetosphere, etc. The main feature of these objects is the existence of a homogeneous (or regular) main media, "matrix," and a system of multiscaled obstacles imbedded in the matrix (drops forming the clouds, cracks in Earth lithosphere, vertices, dislocations, etc.). Fundamental problems appear when someone describes the physical processes in the interface (scattering of acoustic or electromagnetic waves by the clouds, propagation of the seismic waves through the lithosphere, generation of the magnetic field on the solar wind on the surface of the Sun, absorption of high-frequency vibrations or heat energy by thin coverings with multiscaled inclusions and so forth).

Let $d=3$. We single out three broad and natural types of fractal boundaries: cabbage type, bubble type and web type. A cabbage type fractal contains a countable system of smooth 2-dimensional "cracks" which converge to the outer boundary of the domain. A typical cross section is given in Fig. 2. The exact definition can be found in Sect. 2. A bubble type fractal is a domain with the smooth boundary without a countable set of balls. A 2-dimensional analogue is given in Fig. 3. Web fractals are smooth domains without a countable system of "almost parallel" tubes. A typical cross section transversal to axis of tubes is given in the same Fig. 3. In this paper we consider only a very special type of web fractals: the direct product of a 2-D bubble type domain and an interval. Then the problem can be reduced to the 2-dimensional problem for bubble fractals.

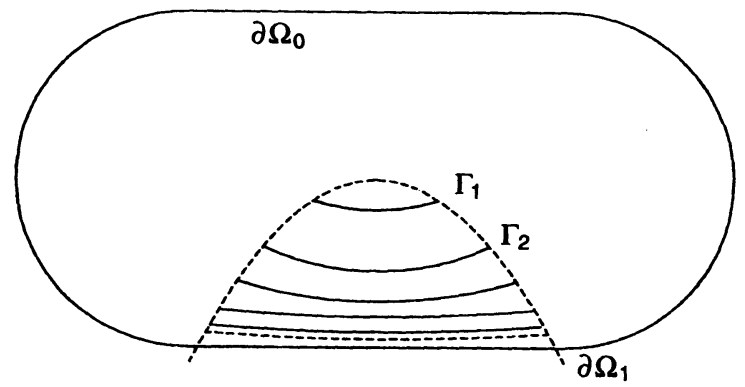

Fig. 2 


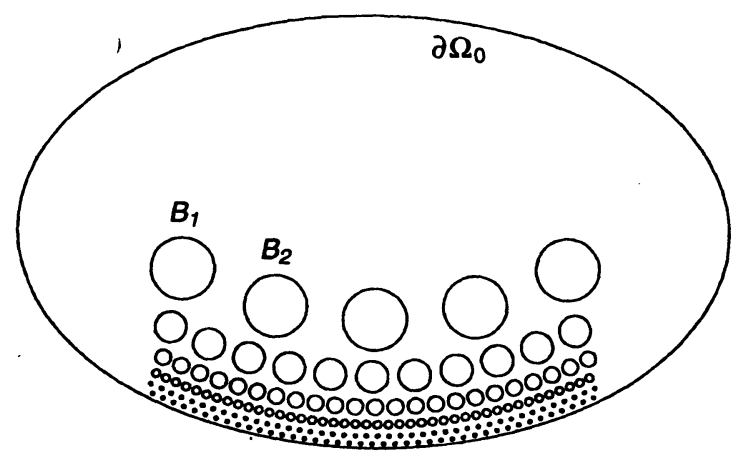

Fig. 3

For cabbage type domains the boundary has fractal structure in the normal direction only. We show that in this case the spectral dimension $s$ coincides with the Minkowski dimension $m$, and moreover under some natural assumptions the MWB conjecture is valid. In the other two cases the MWB conjecture fails. In fact, the answer depends on the electrostatic properties of the boundary. In bubble type domains the spectral dimension $s$ depends on the Newton capacity of the bubbles, in web type domains it depends on the logarithmic capacity of the circles in a crosssection of the web. In particular we show that if radii of the bubbles (or circles in the cross-section of the web) are decreasing fast enough then $s=d-1$, and at the same time the Minkowski dimension can be an arbitrary number between $d-1$ and $d$.

Let us mention that in the case of a smooth boundary the second term in (9) depends on mes $(\partial \Omega)$. It leads to the assumption that formula (9) with $s=d-1$ could be valid for bubble type domains if $\sum r_{i}^{d-1}<\infty$. However it is not true. In Sect. 3 we show that $s=d-1$ if $\sum r_{i}^{d-2}<\infty$. In our next article we will show that $s$ may not be equal to $d-1$ if $\sum r_{i}^{d-1}<\infty$, but $\sum r_{i}^{d-2}=\infty$.

Remark 1. In fact we find the second term of asymptotics only for the integral transformation (2) or (3) of $N^{-}(\lambda)$. It gives some information about the asymptotics of $N^{-}(\lambda)$, and in particular it gives the exact value of the second term of spectral asymptotics of $N^{-}(\lambda)$ under the assumption that this term exists (i.e. the spectral dimension is defined).

\section{Cabbage Type Domains}

We will start this section by recalling the definition of the Minkowski dimension of the boundary $\partial \Omega$ of an open set $\Omega \subset \Re^{d}$. Then we prove the Minkowski measurability of cabbage type domains and find the asymptotic behavior of the $\Theta$-function for the Dirichlet Laplacian in these domains.

Definition 1. Let $(\partial \Omega)_{\varepsilon}$ be an $\varepsilon$-neighborhood of the boundary and $\operatorname{mes}(\partial \Omega)_{\varepsilon}^{+}$be the Lebesgue measure of the interior part $(\partial \Omega)_{\varepsilon}^{+}=(\partial \Omega)_{\varepsilon} \cap \Omega$ of this neighborhood. We say that $\partial \Omega$ is Minkowski measurable if there is a constant $m$ such that 
$\operatorname{mes}(\partial \Omega)_{\varepsilon}^{+} / \varepsilon^{d-m}$ has a nonzero limit as $\varepsilon \rightarrow 0$. The constant $m=m(\partial \Omega)$ is called the Minkowski dimension of $\partial \Omega$, and the limit

$$
|\partial \Omega|_{m}:=\lim _{\varepsilon \rightarrow+0} \frac{\operatorname{mes}(\partial \Omega)_{\varepsilon}^{+}}{\varepsilon^{d-m}}
$$

is called the Minkowski content of $\partial \Omega$.

For example, if $\Omega$ is a domain with a compact smooth boundary, then $m(\partial \Omega)=$ $d-1$ and the Minkowski content is equal to the surface area of $\partial \Omega$. If $\Omega=\Re^{d} \backslash\{0\}$ and $\partial \Omega$ consists of one point, then $m(\partial \Omega)=0$ and the Minkowski content is equal to the volume of the unit $d$-dimensional ball.

Now we give a definition of domains with fractal boundaries of cabbage type. Let domains $\Omega_{0}, \Omega_{1} \subset \Re^{d}$ be given by equations $\Omega_{0}=\{x: F(x)>0\}, \Omega_{1}=$ $\{x: G(x)>0\}$, where $F, G: \Re^{d} \rightarrow \Re, d \geqq 1$, are smooth functions (at least $C^{2}$ class) without critical points at their zero level sets: $\nabla F(x) \neq 0$ as $F(x)=0$, $\nabla G(x) \neq 0$ as $G(x)=0$. We assume that the boundary $\partial \Omega_{0}$ is compact and oneconnected, and $|\nabla F(x)|>\gamma>0$ as $|F(x)| \leqq 1$. In this case the level sets $\{x$ : $F(x)=\varepsilon\},|\varepsilon| \leqq 1$ will be smooth one-connected surfaces. Let domains $\Omega_{0}, \Omega_{1}$ have nonempty intersection and their boundaries be transversal, or $\overline{\Omega_{0}} \subset \Omega_{1}$.

Definition 2. We say that the domain $\Omega$ has a fractal boundary of cabbage type if $\Omega$ has the form (see Fig. 2):

$$
\Omega=\Omega_{0} \backslash \bigcup_{n=1}^{\infty} \Gamma_{n}
$$

where

$$
\Gamma_{n}=\left\{x: F(x)=n^{-\alpha}, x \in \Omega_{1}\right\}
$$

with an arbitrary fix positive constant $\alpha>0$.

Theorem 3. If $\Omega$ has a cabbage type boundary, then $\partial \Omega$ is Minkowski measurable with

$$
\begin{gathered}
m(\partial \Omega)=d-1+\frac{1}{1+\alpha}, \\
|\partial \Omega|_{m}=c(\alpha) \int_{\Gamma}|\nabla F(\sigma)|^{-\frac{1}{1+\alpha}} d \sigma, \quad \Gamma=\partial \Omega_{0} \cap \Omega_{1}, \quad c(\alpha)=(2 / \alpha)^{\frac{\alpha}{1+\alpha}}(1+\alpha) .
\end{gathered}
$$

Proof. We fix some $\delta$ from the interval $\left(0, \frac{1}{2(1+\alpha)}\right)$ and a very small $\varepsilon>0$. Then we represent $\partial \Omega$ in the form of four nonintersecting sets:

$$
G_{0}=\partial \Omega_{0}, \quad G_{1}=\bigcup_{n>n_{1}} \Gamma_{n}, \quad G_{2}=\bigcup_{n<n_{2}} \Gamma_{n}, \quad G_{3}=\bigcup_{n_{1} \geqq n \geqq n_{2}} \Gamma_{n},
$$

where

$$
n_{1}=n_{1}(\varepsilon)=\varepsilon^{-\frac{1}{1+\alpha}-\frac{\delta}{\alpha}}, \quad n_{2}=n_{2}(\varepsilon)=\varepsilon^{-\frac{1}{1+\alpha}+\delta} .
$$

For any set $Q$ we denote the $\varepsilon$-neighborhood of $Q$ by $(Q)_{\varepsilon}$. Since $\partial \Omega_{0}$ is smooth and compact we have

$$
\operatorname{mes}\left(G_{0}\right)_{\varepsilon} \leqq C \varepsilon .
$$


Let $\tilde{\Gamma}_{n}=\left\{x: F(x)=n^{-\alpha}\right\}$ (and therefore, $\Gamma_{n}=\tilde{\Gamma}_{n} \cap \Omega_{1}$ ). From the fact that $|\nabla F(x)|>\gamma>0$ when $|F(x)| \leqq 1$ it follows that the distance $\rho=\rho\left(x, \partial \Omega_{0}\right)$ between any point $x \subset \tilde{\Gamma}_{n}$ and $\partial \Omega_{0}=\{x: F(x)=0\}$ does not exceed $\gamma^{-1} n^{-\alpha}$ :

$$
\rho\left(x, \partial \Omega_{0}\right) \leqq \gamma^{-1} n^{-\alpha} \quad \text { for } x \in \tilde{\Gamma}_{n}, n \geqq 1 .
$$

Thus $\left(G_{1}\right)_{\varepsilon}$ is located inside the $h_{\varepsilon}$-neighborhood of $\partial \Omega_{0}$ with

$$
h_{\varepsilon}=\gamma^{-1} n^{-\alpha}+\varepsilon=\gamma^{-1} \varepsilon^{\frac{\alpha}{1+\alpha}+\delta}+\varepsilon \leqq C \varepsilon^{\frac{\alpha}{1+\alpha}+\delta},
$$

(the last inequality follows from the fact that $\delta<1 /(1+\alpha)$ ) and therefore

$$
\operatorname{mes}\left(G_{1}\right)_{\varepsilon} \leqq C \varepsilon^{\frac{\alpha}{1+\alpha}+\delta} .
$$

Let us estimate mes $\left(G_{2}\right)_{\varepsilon}$. The measure of the $\varepsilon$-neighborhood of $\Gamma_{n}$ does not exceed $C \varepsilon$ with a constant $C$ independent of $n$. Since $G_{2}$ consists of at most $\left[n_{2}\right]$ surfaces $\Gamma_{n}$, we have:

$$
\operatorname{mes}\left(G_{2}\right)_{\varepsilon} \leqq C \varepsilon \cdot \varepsilon^{-\frac{1}{1+\alpha}+\delta}=C \varepsilon^{\frac{\alpha}{1+\alpha}+\delta} .
$$

Thus

$$
\operatorname{mes}\left(G_{0}\right)_{\varepsilon}+\operatorname{mes}\left(G_{1}\right)_{\varepsilon}+\operatorname{mes}\left(G_{2}\right)_{\varepsilon} \leqq C \varepsilon^{\frac{\alpha}{1+\alpha}+\delta} .
$$

Now we will show that

$$
\operatorname{mes}\left(G_{3}\right)_{\varepsilon} \sim \text { const. } \cdot \varepsilon^{\frac{\alpha}{1+\alpha}} \quad \text { as } \varepsilon \rightarrow+0 .
$$

From (12) it follows that

$$
\rho_{\varepsilon}:=\max _{x \in\left(G_{3}\right)_{\varepsilon}} \rho\left(x, \partial \Omega_{0}\right) \leqq C n_{2}^{-\alpha}+\varepsilon=C \varepsilon^{\frac{\alpha}{1+\alpha}-\alpha \delta}+\varepsilon \leqq C \varepsilon^{\frac{\alpha}{2(\alpha+1)}+\delta_{1}}, \quad \delta_{1}>0 .
$$

The last relation in (14) is valid due to the inequality $\delta<1 / 2(\alpha+1)$.

We denote by $l(\sigma)$ the ray emitted from $\sigma \in \partial \Omega_{0}$ in the direction of the internal normal to $\partial \Omega_{0}$ :

$$
l(\sigma)=\left\{x: x=\sigma+\frac{\nabla F(\sigma)}{|\nabla F(\sigma)|} l, l \geqq 0\right\} .
$$

Since the angle $\theta$ between $l(\sigma)$ and $\nabla F(x)$ at the point $x \in l(\sigma)$ is a smooth function of $x$, and this angle is $\pi / 2$ at $\partial \Omega_{0}$, we have $\theta=\pi / 2+O(\rho)$ for $\rho\left(x, \partial \Omega_{0}\right) \leqq 1$. Together with (12) it gives

$$
\theta=\pi / 2+O\left(n^{-\alpha}\right) \text { for } x \in \tilde{\Gamma}_{n}, n \geqq n_{2} .
$$

Let $\Gamma^{\prime}=\partial \Omega_{0} \cap \Omega_{1}$ be the edge of $\Gamma, \Gamma^{-}$be $A \rho_{\varepsilon}$-constriction of $\Gamma$ and $\Gamma^{+}$be $A \rho_{\varepsilon}$-extension of $\Gamma$ on $\partial \Omega_{0}$, i.e. $\Gamma^{-}=\left\{x: x \in \Gamma, \rho\left(x, \Gamma^{\prime}\right)>A \rho_{\varepsilon}\right\}, \Gamma^{+}$consists of $\Gamma$ and all points $x \in \partial \Omega_{0}$ such that $\rho\left(x, \Gamma^{\prime}\right)<A \rho_{\varepsilon}$. Here $A$ is a big enough constant which will be chosen a couple of lines below. Let

$$
\left(G_{3}\right)_{\varepsilon}^{ \pm}=\left\{x: x \in \bigcup_{n_{1} \geqq n \geqq n_{2}}\left(\tilde{\Gamma}_{n}\right)_{\varepsilon}, x \in l(\sigma) \text { with some } \sigma \in \Gamma^{ \pm}\right\},
$$




$$
\left(G_{3}\right)_{\varepsilon}^{0}=\left\{x: x \in \bigcup_{n_{1} \geqq n \geqq n_{2}}\left(\tilde{\Gamma}_{n}\right)_{\varepsilon}, x \in l(\sigma) \text { with some } \sigma \in \Gamma\right\} .
$$

The transversality of $\partial \Omega_{0}$ and $\partial \Omega_{1}$ together with (14), (16) imply that $\left(G_{3}\right)_{\varepsilon}^{-} \subset$ $\left(G_{3}\right)_{\varepsilon} \subset\left(G_{3}\right)_{\varepsilon}^{+}$if $A$ is big enough. The surface area of $\Gamma^{+} \backslash \Gamma^{-}$does not exceed $C \rho_{\varepsilon}$, and the thicknesses of $\left(G_{3}\right)_{\varepsilon}$ does not exceed $\rho_{\varepsilon}$ (see (14)). Together it leads to the following relations:

$$
\operatorname{mes}\left(G_{3}\right)_{\varepsilon}=\operatorname{mes}\left(G_{3}\right)_{\varepsilon}^{-}+O\left(\left(\rho_{\varepsilon}\right)^{2}\right)=\operatorname{mes}\left(G_{3}\right)_{\varepsilon}^{+}+O\left(\left(\rho_{\varepsilon}\right)^{2}\right) .
$$

From here and the inclusions $\left(G_{3}\right)_{\varepsilon}^{-} \subset\left(G_{3}\right)_{\varepsilon}^{0} \subset\left(G_{3}\right)_{\varepsilon}^{+}$we get that mes $\left(G_{3}\right)_{\varepsilon}=$ $\operatorname{mes}\left(G_{3}\right)_{\varepsilon}^{0}+O\left(\left(\rho_{\varepsilon}\right)^{2}\right)$ and therefore

$$
\operatorname{mes}\left(G_{3}\right)_{\varepsilon}=\int_{\left(G_{3}\right)_{\varepsilon}^{0}} d x+O\left(\varepsilon^{\frac{\alpha}{1+\alpha}+2 \delta_{1}}\right), \quad \delta_{1}>0, \varepsilon \rightarrow+0 .
$$

According to (14) the layers $\left(\tilde{\Gamma}_{n}\right)_{\varepsilon}, n \geqq n_{2}$, are close to $\partial \Omega_{0}$. Hence one can rewrite the last integral as a repeated one and integrate first along $l(\sigma)$, and then along $\Gamma$. From $(16)$ it follows that the intersections $l(\sigma)$ with the layers $\left(\tilde{\Gamma}_{n}\right)_{\varepsilon}$, $n \geqq n_{2}$ are intervals $\Delta=\Delta(n, \sigma, \varepsilon)$ with the distances between $\sigma^{\prime}=l(\sigma) \cap \tilde{\Gamma}_{n}$ and the ends of $\Delta$ equal to $\varepsilon\left(1+O\left(\rho_{\varepsilon}\right)\right)$. We will use the values of $F$ as coordinates along $l(\sigma)$. Let $d \sigma$ be the element of the surface area of $\Gamma$. Then we have

$$
\operatorname{mes}\left(G_{3}\right)_{\varepsilon}=\int_{\Gamma}\left[\int_{U^{\prime} \Delta(n, \sigma, \varepsilon)} J d F\right] d \sigma+o\left(\varepsilon^{\frac{\alpha}{1+\alpha}}\right), \quad J=\left|\frac{d x}{d F d \sigma}\right|,
$$

where $\cup^{\prime} \Delta$ means the union of intervals $\Delta$ for $n_{1} \geqq n \geqq n_{2}$.

Since $J=\frac{1}{|\nabla F(\sigma)|}$ at $\sigma \in \partial \Omega_{0}$ and the Jacobian $J$ is a smooth function, we have $J=\frac{1}{|\nabla F(\sigma)|}+O\left(\rho_{\varepsilon}\right)$ when $\rho\left(x, \partial \Omega_{0}\right) \leqq \rho_{\varepsilon}$. Thus

$$
\operatorname{mes}\left(G_{3}\right)_{\varepsilon}=\int_{\Gamma}\left[\int_{U^{\prime} \Delta(n, \sigma, \varepsilon)}\left(\frac{1}{|\nabla F(\sigma)|}+O\left(\rho_{\varepsilon}\right)\right) d F\right] d \sigma+o\left(\varepsilon^{\frac{\alpha}{1+\alpha}}\right) .
$$

From here and the fact that the increment of $F$ on $l(\sigma)$ in the $\rho_{\varepsilon}$-neighborhood of $\partial \Omega_{0}$ is less than $C \rho_{\varepsilon}$, it follows that

$$
\begin{aligned}
\operatorname{mes}\left(G_{3}\right)_{\varepsilon} & =\int_{\Gamma}\left[\int_{U^{\prime} \Delta(n, \sigma, \varepsilon)} \frac{1}{|\nabla F(\sigma)|} d F\right] d \sigma+o\left(\varepsilon^{\frac{\alpha}{1+\alpha}}\right) \\
& =\int_{\Gamma} \frac{1}{|\nabla F(\sigma)|}\left[\int_{U^{\prime} \Delta(n, \sigma, \varepsilon)} d F\right] d \sigma+o\left(\varepsilon^{\frac{\alpha}{1+\alpha}}\right) .
\end{aligned}
$$

Now we are going to evaluate the internal integral in (21). From (16) it follows that the derivative $\partial F / \partial l$ of $F$ in the direction of $l(\sigma)$ is equal to $|\nabla F(x)|(1+$ $\left.O\left(\rho_{\varepsilon}\right)\right)$ at points $x \in l(\sigma)$ with $\rho\left(x, \partial \Omega_{0}\right) \leqq \rho_{\varepsilon}$. The smoothness of $\nabla F$ implies that $|\nabla F(x)|=|\nabla F(\sigma)|\left(1+O\left(\rho_{\varepsilon}\right)\right)$ for $x \in l(\sigma), \rho\left(x, \partial \Omega_{0}\right) \leqq \rho_{\varepsilon}$. Thus

$$
\frac{\partial F}{\partial l}=|\nabla F(\sigma)|\left(1+O\left(\rho_{\varepsilon}\right)\right) \text { for } x \in l(\sigma), \rho\left(x, \partial \Omega_{0}\right) \leqq \rho_{\varepsilon} .
$$


By integrating $\frac{\partial F}{\partial l}$ along $l(\sigma)$ and taking into account that $F\left(\sigma^{\prime}\right)=n^{-\alpha}$ we get

$$
F(x)=n^{-\alpha} \pm \varepsilon|\nabla F(\sigma)|\left(1+O\left(\rho_{\varepsilon}\right)\right)
$$

at the end points $x$ of $\Delta(n, \sigma, \varepsilon)$. Let us replace the intervals $\Delta(n, \sigma, \varepsilon)$ in (21) by the close intervals $\tilde{\Delta}(n, \sigma, \varepsilon)$ such that $F(x)=n^{-\alpha} \pm \varepsilon|\nabla F(\sigma)|$ at the end points of $\tilde{\Delta}$. Since the union $\cup^{\prime} \Delta$ consists of at most $n_{2}(\varepsilon)$ intervals, the error will have an order $O\left(n_{2}(\varepsilon) \cdot \varepsilon \rho_{\varepsilon}\right)=o\left(\varepsilon^{\frac{\alpha}{1+\alpha}}\right)$. Thus

$$
\operatorname{mes}\left(G_{3}\right)_{\varepsilon}=\int_{\Gamma} \frac{1}{|\nabla F(\sigma)|}\left[\int_{U^{\prime}(n, \sigma, \varepsilon)} d F\right] d \sigma+o\left(\varepsilon^{\frac{\alpha}{1+\alpha}}\right) .
$$

Now the internal integral is equal to the length of the system of intervals

$$
\left[n^{-\alpha}-\varepsilon|\nabla F(\sigma)|, n^{-\alpha}+\varepsilon|\nabla F(\sigma)|\right], \quad n_{1} \geqq n \geqq n_{2}
$$

on the $F$-axis. Let us find $n=n^{*}$ from the equation

$$
n^{-\alpha}-\varepsilon|\nabla F(\sigma)|=(n+1)^{-\alpha}+\varepsilon|\nabla F(\sigma)| .
$$

It is obvious that

$$
n^{*} \sim\left[\frac{\alpha}{2 \varepsilon|\nabla F(\sigma)|}\right]^{\frac{1}{1+\alpha}}, \quad \varepsilon \rightarrow+0 .
$$

The intervals with $n>n^{*}$ intersect each other and cover the segment

$$
\left[\left(n_{1}(\varepsilon)\right)^{-\alpha}-\varepsilon|\nabla F(\sigma)|,\left(n^{*}-1\right)^{-\alpha}+\varepsilon|\nabla F(\sigma)|\right] .
$$

The length $h$ of this segment has order $\left(n^{*}\right)^{-\alpha}$, i.e.

$$
h \sim\left[\frac{2 \varepsilon|\nabla F(\sigma)|}{\alpha}\right]^{\frac{\alpha}{1+\alpha}}, \quad \varepsilon \rightarrow+0 .
$$

The intervals with $n \in\left[n_{2}, n^{*}\right]$ do not intersect each other, and their common length is

$$
2 \varepsilon|\nabla F(\sigma)|\left(n^{*}-n_{2}\right) \sim(2 \varepsilon|\nabla F(\sigma)|)^{\frac{\alpha}{1+\alpha}} \alpha^{\frac{1}{1+\alpha}}, \quad \varepsilon \rightarrow+0 .
$$

Thus the internal integral in (22) is equal to

$$
\left[\frac{2 \varepsilon|\nabla F(\sigma)|}{\alpha}\right]^{\frac{\alpha}{1+\alpha}}(1+\alpha)+o\left(\varepsilon^{\frac{\alpha}{1+\alpha}}\right), \quad \varepsilon \rightarrow+0 .
$$

Together with (22) and (13) it gives (10), (11). Theorem 3 is proved.

The next theorem gives the asymptotic behavior of the $\theta$-function (2) for the Dirichlet Laplacian $-\Delta^{-}$in domains with fractal boundaries of cabbage type. Before we formulate this theorem we give the corresponding 1-dimensional result which follows from [La2] and will be used in an essential way to prove the theorem.

Let us recall that we denote by $l(\sigma)$ the ray emitted from $\sigma \in \partial \Omega_{0}$ in the direction of the internal normal to $\partial \Omega_{0}$ (see (15)). Let $l^{\prime}(\sigma)$ be the maximal segment of $l(\sigma)$ with the beginning point at $\sigma$ on which $0 \leqq F(x) \leqq 1$ and $\left|l^{\prime}(\sigma)\right|$ be the length of $l^{\prime} \sigma$ ). All points of $l^{\prime} \sigma$ ), except $\sigma$, belong to the interior of $\Omega_{0}$ because $|\nabla F(x)| \neq 0$ when $0 \leqq F(x) \leqq 1$ (and $F>0$ on $\Omega_{0}, F=0$ on $\partial \Omega_{0}$ ). We will use 
$s=|x-\sigma|$ as the coordinates of points $x$ on $l(\sigma)$. We denote by $\left\{l_{n}\right\}$ the system of subintervals $l_{n}$ on $l^{\prime}(\sigma)$ determined by inequalities $(n+1)^{-\alpha}<F(x)<n^{-\alpha}, n=$ $1,2, \ldots$ Let $P\left(t, s, s^{\prime}\right)$ be the 1-dimensional Green function for the heat equation on $\left\{l_{n}\right\}$ with Dirichlet boundary conditions at end points of all subintervals, and $\widetilde{P}=P-(4 \pi t)^{-1 / 2} \exp \left(-\frac{\left|s-s^{\prime}\right|^{2}}{4 \pi t}\right)$.

We denote by $\zeta(z)$ the classical Riemann function which is equal to $\varsigma(z)=$ $\sum j^{-z}$ for $\operatorname{Re} z>1$ and is determined as a meromorphic extension for other $z$. In particular, $\varsigma(z)=\frac{1}{z-1}+\int_{1}^{\infty}\left([t]^{-z}-t^{-z}\right) d t$ for $0<z<1$. Let $\Gamma(z)=\int_{0}^{\infty} e^{-t} t^{z-1} d t$, $\operatorname{Re} z>0$, be the Gamma function.

Lemma 4. The following formulas are valid when $t \rightarrow 0$ :

$$
\begin{aligned}
\int_{l^{\prime}(\sigma)} P(t, s, s) d s= & \frac{\left|l^{\prime}(\sigma)\right|}{2 \sqrt{\pi}} t^{-\frac{1}{2}}-\frac{1}{2(1+\alpha)}\left(\frac{\alpha}{\pi|\nabla F(\sigma)|}\right)^{\frac{1}{1+\alpha}}\left(-\varsigma\left(\frac{1}{1+\alpha}\right)\right) \\
& \times \Gamma\left(\frac{1}{2(1+\alpha)}\right) t^{-\frac{1}{2(1+\alpha)}}(1+o(1))
\end{aligned}
$$

and respectively

$$
\begin{aligned}
\int_{l^{\prime}(\sigma)} \widetilde{P}(t, s, s) d s= & -\frac{1}{2(1+\alpha)}\left(\frac{\alpha}{\pi|\nabla F(\sigma)|}\right)^{\frac{1}{1+\alpha}}\left(-\varsigma\left(\frac{1}{1+\alpha}\right)\right) \\
& \times \Gamma\left(\frac{1}{2(1+\alpha)}\right) t^{-\frac{1}{2(1+\alpha)}}(1+o(1)),
\end{aligned}
$$

where the estimates of remainders are uniform with respect to $\sigma \subset \partial \Omega_{0}$.

Proof. Let $D \subset \Re$ consist of a system of nonintersecting intervals such that their lengths $\left|l_{j}\right|$ have an order $\left|l_{j}\right| \sim L j^{-(1+\alpha)}$ with $\alpha>0$ as $j \rightarrow \infty$. Then [La2] $D$ is Minkowski measurable, $m(\partial D)=\frac{1}{1+\alpha},|\partial D|_{m}=2^{1-m}(1-m)^{-1} L^{m}$, and the counting function of the corresponding 1-dimensional Laplacian with Dirichlet boundary conditions at the ends of intervals $l_{j}$ has the following asymptotic behavior:

$$
N^{-}(\lambda)=\frac{1}{\pi}|D| \sqrt{\lambda}-2^{m-1} \pi^{-m}(1-m)(-\varsigma(m))|\partial D|_{m} \lambda^{m / 2}(1+o(1)) \quad \text { as } \lambda \rightarrow \infty \text {. }
$$

The assertion of the lemma follows from the last formula applied to $D=L^{\prime}(\sigma)$ $\left(L=\frac{\alpha}{|\nabla F(\sigma)|}\right.$ in this case) and from (2).

Theorem 5. Let domain $\Omega$ have a fractal boundary of a cabbage type with parameter $\alpha>0$. Then $\theta^{-}$-function has the following asymptotics as $t \rightarrow 0$ :

$$
\theta^{-}(t):=\int_{0}^{\infty} e^{-\lambda t} d N^{-}(\lambda)=\frac{|\Omega|}{(4 \pi t)^{d / 2}}+c(d, m) \frac{|\partial \Omega|_{m}}{t^{m / 2}}(1+o(1)),
$$

where $m=m(\partial \Omega)$ and $|\partial \Omega|_{m}$ are defined in Theorem 1 , and

$$
\begin{aligned}
c(d, m) & =\frac{\alpha}{(1+\alpha)^{2}} 2^{-d-\frac{\alpha}{1+\alpha}} \pi^{-\frac{d}{2}-\frac{1}{1+\alpha}}\left(-\varsigma\left(\frac{1}{1+\alpha}\right)\right) \Gamma\left(\frac{1}{2(1+\alpha)}\right) \\
& =(m-d+1)(d-m) 2^{m-2 d} \pi^{\frac{d-1}{2}-m}(-\varsigma(m-d+1)) \Gamma\left(\frac{m-d+1}{2}\right) .
\end{aligned}
$$


Remark 2. It means that the MWB-conjecture is valid for cabbage type fractals (in a weaker sense: for $\theta^{-}$-function).

Proof. Let $p_{0}(t, x, y)$ be the Green function of the heat equation in $\Re^{d}$ :

$$
p_{0}=\frac{1}{(4 \pi t)^{d / 2}} e^{-\frac{|x-y|^{2}}{4 \pi t}}
$$

and $p=p(t, x, y)$ be the Green function of the corresponding Dirichlet problem in $\Omega$ (we drop the upper index in the notation of the Green function which was used earlier to distinguish Dirichlet and Neumann problems because now we are dealing only with the Dirichlet problem):

$$
\frac{\partial p}{\partial t}=\Delta p, \quad x \in \Omega ; \quad p=0, \quad x \in \partial \Omega ; \quad p=\delta(x-y), \quad t=0 .
$$

Let $\widetilde{p}=p-p_{0}$. Then

$$
\frac{\partial \widetilde{p}}{\partial t}=\Delta \widetilde{p}, \quad x \in \Omega ; \quad \widetilde{p}=-p_{0}, \quad x \in \partial \Omega ; \quad \tilde{p}=0, \quad t=0 .
$$

From $p_{0}(t, x, x)=(4 \pi t)^{-d / 2}$ and (2) it follows that

$$
\theta^{-}(t)=\int_{\Omega} p(t, x, x) d x=|\Omega|(4 \pi t)^{-d / 2}+\int_{\Omega} \tilde{p}(t, x, x) d x .
$$

We fix a small $t_{0}>0$ and take $\varepsilon=t_{0}^{1 / 2-v}$ with a small $v>0$ which will be chosen later. We represent $\Omega$ in the form $U_{1} \cup U_{2} \cup U_{3}$ where

$$
\begin{gathered}
U_{1}=\left\{x: x \in \Omega, F(x)<n_{1}^{-\alpha}, n_{1}=n_{1}(\varepsilon)=\varepsilon^{-\frac{1}{1+\alpha}-\frac{\delta}{\alpha}}\right\}, \\
U_{2}=\left\{x: x \in \Omega, F(x)>n_{2}^{-\alpha}, n_{2}=n_{2}(\varepsilon)=\varepsilon^{-\frac{1}{1+\alpha}+\delta}\right\}, \\
U_{3}=\left\{x: x \in \Omega, n_{1}^{-\alpha} \leqq F(x) \leqq n_{2}^{-\alpha}\right\}
\end{gathered}
$$

with a small positive $\delta$ such that

$$
0<\delta<\min \left(\frac{1}{2(1+\alpha)}, \frac{\alpha^{2}}{\left(\alpha^{2}+\alpha+1\right)(1+\alpha)}\right) .
$$

We will estimate the integrals $\int|\tilde{p}(t, x, x)| d x$ over each of domains $U_{j}, j=$ $1,2,3$, separately. We will show that the integral over $U_{1}$ does not contribute to the asymptotics of $\theta^{-}(t)$ because the mes $U_{1}$ is small $\left(U_{1}\right.$ is located inside a very thin neighborhood of $\partial \Omega_{0}$ ). The integral over $U_{2}$ does not contribute to the asymptotics of $\theta^{-}(t)$ because $U_{2}$ contains only a small part of the boundary $\partial \Omega$, and the main contribution to the asymptotics will be given by the integral over $U_{3}$.

We will need the following two estimates. The first one is an obvious consequence of the maximum principle:

$$
|\widetilde{p}(t, x, y)| \leqq p_{0}(t, x, y) \leqq(4 \pi t)^{-d / 2}
$$

Now let us note that $p_{0} \leqq C t$ if $t \leqq t_{0},|x-y| \geqq \varepsilon=t_{0}^{1 / 2-v}$ (in fact, $p_{0}$ decays exponentially as $t$ goes to zero). From here and the fact that for any domain $Q$ the 
Green function $p$ of the problem in $Q$ does not exceed $p_{0}$ we get

$$
0 \leqq p(t, x, y) \leqq C t \text { when }|x-y| \geqq \varepsilon=t_{0}^{1 / 2-v}, t \leqq t_{0} \leqq 1,
$$

where the constant $C$ does not depend on the domain $Q$.

From (12) it follows that mes $U_{1} \leqq C n_{1}^{-\alpha}=C \varepsilon^{\frac{\alpha}{1+\alpha}+\delta} \leqq C t_{0}^{\frac{\alpha}{2(1+\alpha)}+\frac{\delta}{4}}$ if $v$ is small enough $\left(v\left(\frac{1}{1+\alpha}+\delta\right) \leqq \frac{\delta}{4}\right)$. Together with (29) it gives the estimate:

$$
\int_{U_{1}}|\tilde{p}(t, x, x)| d x \leqq C t^{-d / 2} t_{0}^{\alpha / 2(\alpha+1)+\delta / 4}, \quad 0<t \leqq t_{0} \leqq 1 .
$$

Before estimating the integral over $U_{2}$ let us recall that $p_{0}(x, y, t) \leqq C t$ when $x \in \partial \Omega, \rho(y, \partial \Omega) \geqq \varepsilon, t \leqq t_{0} \leqq 1$. From here and the maximum principle applied to (26) it follows that $|\widetilde{p}|<C t$ for $x \in \Omega, 0<t \leqq t_{0} \leqq 1$ if $\rho(y, \partial \Omega) \geqq \varepsilon$. The constant $C$ here is independent of $x, t, t_{0}, y$. Hence

$$
\int_{\rho(x, \partial \Omega) \geqq \varepsilon}|\widetilde{p}(t, x, x)| d x \leqq C t \quad \text { for } 0<t \leqq t_{0} .
$$

with a constant $C$ independent of $t, t_{0}$. Now we represent $U_{2}$ in the form $U_{2}^{\prime} \cup U_{2}^{\prime \prime}$, where $U_{2}^{\prime}$ is the set of points $x \in U_{2}$ for which $\rho(x, \partial \Omega) \geqq \varepsilon, U_{2}^{\prime \prime}=U_{2} \backslash U_{2}^{\prime}$. The same estimate is valid for mes $U_{2}^{\prime \prime}$ as for mes $U_{1}$ (see proof of (13)) and therefore (31) is true for the integral over $U_{2}^{\prime \prime}$. Together with the estimate (32) for the integral over $U_{2}^{\prime}$ it implies that

$$
\int_{U_{2}}|\widetilde{p}(t, x, x)| d x \leqq C t^{-d / 2} t_{0}^{\alpha / 2(\alpha+1)+\delta / 4}, \quad 0<t \leqq t_{0} .
$$

Domain $U_{3}$ is located inside a thin neighborhood of $\partial \Omega_{0}$, whose thickness $d_{\varepsilon}$ can be estimated due to (12) as follows:

$$
d_{\varepsilon}:=\max _{x \in U_{3}} \rho\left(x, \partial \Omega_{0}\right) \leqq C n_{2}^{-\alpha}=C \varepsilon^{\frac{\alpha}{1+\alpha}-\alpha \delta} .
$$

On the other hand $F(x) \geqq n_{1}^{-\alpha}=\varepsilon^{\frac{\alpha}{1+\alpha}+\delta}$ when $x \in U_{3}$. Since $\varepsilon \ll 1, \frac{\alpha}{1+\alpha}+\delta<1$ and $|\nabla F(x)|$ is bounded in a neighborhood of $\partial \Omega_{0}$, it implies $\rho\left(U_{3}, \partial \Omega_{0}\right) \geqq \varepsilon$.

We split $U_{3}$ into three parts: $U_{3}=U_{3}^{0} \cup U_{3}^{\prime} \cup U_{3}^{\prime \prime}$, where $U_{3}^{0}$ consists of the points $x \in U_{3}$ belonging to a small neighborhood of $\partial \Omega_{1}\left(\Omega_{1}\right.$ was introduced in the definition of domains of cabbage type $), U_{3}^{\prime}=\left(U_{3} \cap \Omega_{1}\right) \backslash U_{3}^{0}, U_{3}^{\prime \prime}=U_{3} \backslash\left(\Omega_{1} \cup U_{3}^{0}\right)$. To be more exact we take

$$
U_{3}^{0}=\left\{x: x \in U_{3}, x \in l(\sigma) \text { with some } \sigma \in \Gamma^{0}\right\},
$$

where $l(\sigma)$ is determined in (15) and $\Gamma^{0}$ is the set of points $\sigma \in \partial \Omega_{0}$ belonging to the $A d_{\varepsilon}$-neighborhood of $\partial \Omega_{0} \cap \partial \Omega_{1}$. We choose $A$ big enough such that $\left(\partial \Omega_{1}\right)_{\varepsilon}$ does not intersect $U_{3}^{\prime}$ and $U_{3}^{\prime \prime}$. It can be done because $\partial \Omega_{0}$ and $\partial \Omega_{1}$ are transversal, and $\varepsilon \ll d_{\varepsilon}$. Since $\rho\left(U_{3}, \partial \Omega_{0}\right) \geqq \varepsilon$ and $\rho\left(U_{3}^{\prime \prime}, \Omega_{1}\right) \geqq \varepsilon$, estimate (32) is valid for the integral over $U_{3}^{\prime \prime}$. Since $\delta_{1}=\frac{\alpha}{1+\alpha}-2 \alpha \delta>0$ and mes $U_{3}^{0} \leqq C d_{\varepsilon}^{2} \leqq C \varepsilon^{\frac{\alpha}{1+\alpha}+\delta_{1}}$, estimate (31) with $\delta=\delta_{1}$ is valid for the integral over $U_{3}^{0}$. From here, (31) and (33) we get

$$
\int_{\Omega \backslash U_{3}^{\prime}}|\tilde{p}(t, x, x)| d x \leqq C t^{-d / 2} t_{0}^{\alpha / 2(\alpha+1)+\delta^{\prime} / 4}, \quad 0<t \leqq t_{0}, \delta^{\prime}=\min \left(\delta, \delta_{1}\right) .
$$


Now we will evaluate the integral over $U_{3}^{\prime}$. We rewrite this integral as a repeated one and integrate first along $l(\sigma)$ and then along $\Gamma^{\prime}=\Gamma \backslash \Gamma^{0}$ :

$$
\int_{U_{3}^{\prime}}|\widetilde{p}(t, x, x)| d x=\int_{\Gamma^{\prime}}\left[\int_{l(\sigma) \cap U_{3}^{\prime}}|\widetilde{p}(t, x, x)| J^{\prime} d l\right] d \sigma, \quad J^{\prime}=\left|\frac{d x}{d l d \sigma}\right| .
$$

Since the Jacobian $J^{\prime}=1$ at $\sigma \in \partial \Omega_{0}$, the thickness of $U_{3}^{\prime}$ does not exceed $d_{\varepsilon}$ and $J^{\prime}$ is a smooth function, we have $J^{\prime}=1+O\left(d_{\varepsilon}\right)$. From here and (29) it follows that the error will have an order $O\left(t^{-d / 2} d_{\varepsilon} \operatorname{mes} U_{3}^{\prime}\right)=O\left(t^{-d / 2} d_{\varepsilon}^{2}\right)$ if we drop $J^{\prime}$ in (36). Since $d_{\varepsilon}^{2} \leqq C \varepsilon^{\frac{\alpha}{1+\alpha}+\delta_{1}}$, the error does not exceed the right-hand side in (35). Thus (35) and (36) imply

$$
\int_{\Omega}|\widetilde{p}(t, x, x)| d x=\int_{\Gamma^{\prime}}\left[\int_{l(\sigma) \cap U_{3}^{\prime}}|\widetilde{p}(t, x, x)| d l\right] d \sigma+O\left(t^{-\frac{d}{2}} t_{0}^{\frac{\alpha}{2(\alpha+1)}+\frac{\delta^{\prime}}{4}}\right), \quad 0<t \leqq t_{0} .
$$

Our next step is to express $\tilde{p}$ in the right-hand side of (37) through the 1-dimensional Green function for the heat equation on the set $\left\{l_{n}\right\}$ of subintervals $l_{n}$ of the ray $l^{\prime}(\sigma)$ (see Lemma 4) with Dirichlet boundary conditions at points $x_{n}=l^{\prime}(\sigma) \cap \Gamma_{n}$. Formula (37) can be rewritten in the form

$$
\int_{\Omega}|\tilde{p}(t, x, x)| d x=\sum_{n_{1} \geqq n \geqq n_{2}} \int_{\Gamma^{\prime}}\left[\int_{l_{n}}|\tilde{p}(t, x, x)| d l\right] d \sigma+O\left(t^{-\frac{d}{2}} t_{0}^{\frac{\alpha}{2(\alpha+1)}+\frac{\delta^{\prime}}{4}}\right), \quad 0<t \leqq t_{0} .
$$

We cut two small pieces of the length $\delta_{n}=\varepsilon^{(1+\alpha) \delta}\left|l_{n}\right|$ from the ends of the segments $l_{n}$. Here $\left|l_{n}\right|$ is the length of $l_{n}$. We denote by $l_{n}^{-}$the shorter segment. The union (with respect to $n$ and $\sigma \in \Gamma^{\prime}$ ) of all cut off pieces covers a part of $U_{3}^{\prime}$ whose measure does not exceed $C \varepsilon^{(1+\alpha) \delta}$ mes $U_{3}^{\prime} \leqq C \varepsilon^{\frac{\alpha}{1+\alpha}+\delta}$. The last inequality follows from (34). Together with (29) it allows us to replace $l_{n}$ in (38) by $l_{n}^{-}$because the error due to this change does not exceed the remainder term in (38). Thus

$$
\int_{\Omega}|\tilde{p}(t, x, x)| d x=\sum_{n_{1} \geqq n \geqq n_{2}} \int_{\Gamma^{\prime}}\left[\int_{l_{n}^{-}}|\tilde{p}(t, x, x)| d l\right] d \sigma+O\left(t^{-\frac{d}{2}} t_{0}^{\frac{\alpha}{(\alpha+1)}+\frac{\delta^{\prime}}{4}}\right), \quad 0<t \leqq t_{0} .
$$

Let $T_{n}, Q_{n}$ be two planes through the ends of $l_{n}^{-}$which are orthogonal to $l(\sigma)$. Let us show that surfaces $\Gamma_{n}, \Gamma_{n+1}$ do not intersect $T_{n}, Q_{n}$ in an $\varepsilon$-neighborhood of $l(\sigma)$ if $t_{0}$ (and therefore $\varepsilon$ ) is small enough. Since the angles between $l(\sigma)$ and a normal vector to $\Gamma_{n}, \Gamma_{n+1}$ have order $O\left(n^{-\alpha}\right)$ (see (16)) and the curvatures of $\Gamma_{n}$, $\Gamma_{n+1}$ are uniformly bounded, the deviations of these surfaces in the direction of $l(\sigma)$ in an $\varepsilon$-neighborhood of $l(\sigma)$ do not exceed $A\left(\varepsilon n^{-\alpha}+\varepsilon^{2}\right)$ where the constant $A$ does not depend on $n$ and $\sigma$. Thus, we have to check that

$$
\varepsilon^{(1+\alpha) \delta}\left|l_{n}\right| \geqq A\left(\varepsilon n^{-\alpha}+\varepsilon^{2}\right), \quad n \geqq n_{2}
$$

for small enough $\varepsilon>0$. Since $F=n^{-\alpha}$ on $\Gamma_{n}$ and $|\nabla F|$ is bounded, $\left|l_{n}\right|$ has an order $O\left(n^{-\alpha}-(n+1)^{-\alpha}\right)=O\left(n^{-\alpha-1}\right)$ as $n \rightarrow \infty$, and it remains to prove that

$$
\varepsilon^{(1+\alpha) \delta} n^{-\alpha-1} \geqq C\left(\varepsilon n^{-\alpha}+\varepsilon^{2}\right), \quad n \geqq \varepsilon^{-\frac{1}{1+\alpha}+\delta}
$$


if $\varepsilon>0$ is small enough. The last estimate is obvious if $\delta=0$, and therefore it is valid if $\delta$ is small enough. In fact it is not difficult to check that (28) provides (40) (otherwise we could choose a smaller bound for $\delta$ ). Hence it is proved that the surfaces $\Gamma_{n}, \Gamma_{n+1}$ do not intersect $T_{n}, Q_{n}$ in an $\varepsilon$-neighborhood of $l(\sigma)$ if $t_{0}$ is small enough.

We denote by $K_{n}$ the layer between $T_{n}$ and $Q_{n}$, and by $K_{n}^{\varepsilon}$ the right circle cylinder of the radius $\varepsilon$ with axis $l_{n}^{-}$and bases on $T_{n}, Q_{n}$ (see Fig. 4). Let $p_{n}$ be the Green function of the Dirichlet problem for the heat equation in the layer $K_{n}$. Let $y \in l_{n}^{-}$. We are going to compare the values of the Green functions $p=p^{-}$(see (25)) and $p_{n}$ when $x \in K_{n}^{\varepsilon}, y \in l_{n}^{-}$. It is obvious that $p \geqq p_{n}$ on the bases of the cylinder $K_{n}^{\varepsilon}$ because $p \geqq 0$ at $\Omega$ and $p_{n}=0$ on $T_{n}$ and $Q_{n}$. On the lateral surface of the cylinder both Green functions can be estimated as in (30). From here and from the maximum principle applied to the heat equation in $K_{n}^{\varepsilon}$ it follows that

$$
p \geqq p_{n}-C t \text { when } x \in K_{n}^{\varepsilon}, y \in l_{n}^{-}, t \leqq t_{0} .
$$

Since functions $\widetilde{p}=p-p_{0}$ and $\tilde{p}_{n}=p_{n}-p_{0}$ are negative, (41) gives the inequality

$$
\widetilde{p} \leqq \widetilde{p}_{n}+C t \quad \text { when } x \in K_{n}^{\varepsilon}, y \in l_{n}^{-}, t \leqq t_{0} .
$$

Together with (39) it leads to the following estimate:

$$
\int_{\Omega}|\tilde{p}(t, x, x)| d x \leqq \sum_{n_{1} \geqq n \geqq n_{2} \Gamma^{\prime}}\left[\int_{l_{n}^{-}}\left|\tilde{p}_{n}(t, x, x)\right| d l\right] d \sigma+C t^{-\frac{d}{2}} t_{0}^{\frac{\alpha}{2(\alpha+1)}+\frac{\delta^{\prime}}{4}}, \quad 0<t \leqq t_{0} .
$$

We choose a new Euclidean basis in $\Re^{n}$ with the origin at the point $\sigma$ and with coordinates $(s, u), s \in \Re, u \in \Re^{n-1}$, where semiaxes $s \geqq 0$ coincide with $l(\sigma)$. Let us temporarily denote the free space Green function (24) by $p_{0}^{d}$ in order to stress its dependence on the dimension $d$. Let $P_{n}^{-}=P_{n}^{-}\left(t, s, s^{\prime}\right)$ be the 1-dimensional Green function for the segment $l_{n}^{-}$, and $\widetilde{P_{n}^{-}}=P_{n}^{-}-p_{0}^{1}$. The Green function $p_{n}$ in the layer is equal to $p_{0}^{d-1} P_{n}^{-}$, and therefore $\widetilde{p}_{n}(t, x, x)=(4 \pi t)^{(1-d) / 2} \widetilde{P_{n}^{-}}(t, s, s), x=x(s, u)$. If

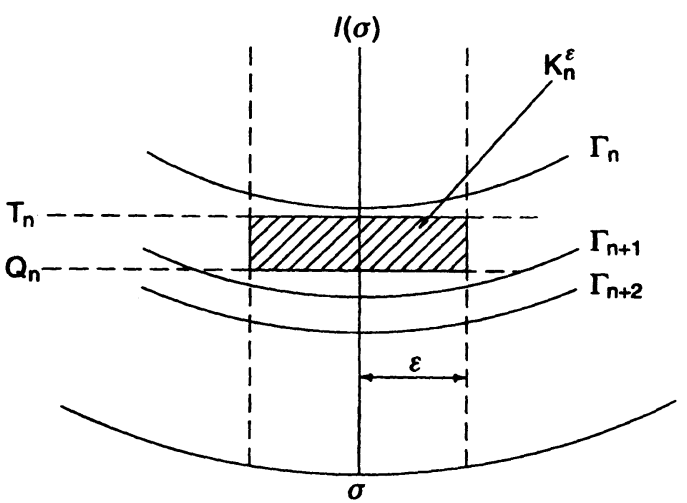

Fig. 4 
we substitute it in the last inequality and put $t=t_{0}$ we will get

$$
(4 \pi t)^{\frac{d-1}{2}} \int_{\Omega}|\tilde{p}(t, x, x)| d x \leqq \sum_{n_{1} \geqq n \geqq n_{2}} \int_{\Gamma^{\prime}}\left[\int_{l_{n}^{-}}\left|\widetilde{P_{n}^{-}}(t, s, s)\right| d s\right] d \sigma+C t^{\frac{-1}{2(1+\alpha)}+\frac{\delta^{\prime}}{4}}, \quad t \rightarrow 0 .
$$

Now we will use the following simple fact: if $q_{a}=q_{a}\left(t, s, s^{\prime}\right)$ is the Green function of the 1-dimensional heat equation on the segment $[0, a]$ then $a q_{a}\left(a^{2} t, a s, a s^{\prime}\right)=$ $q_{1}\left(t, s, s^{\prime}\right)$ and therefore

$$
\int_{0}^{a} q_{a}(t, s, s) d s=\int_{0}^{a} q_{b}\left(\frac{b^{2}}{a^{2}} t, s, s\right) d s .
$$

The same relation is valid for $\tilde{q}_{a}=q_{a}-p_{0}^{1}$. Taking into account that $\left|l_{n}^{-}\right| /\left|l_{n}\right|=$ $1-2 \varepsilon^{(1+\alpha) \delta}=1-2 t^{h}$ with $h=(1+\alpha) \delta(1 / 2-v)>0$ allows us to rewrite $(42)$ in the form

$$
\begin{aligned}
& (4 \pi t)^{\frac{d-1}{2}} \int_{\Omega}|\tilde{p}(t, x, x)| d x \\
& \quad \leqq \sum_{n_{1} \geqq n \geqq n_{2}} \int_{\Gamma^{\prime}}\left[\int_{l_{n}}\left|\widetilde{P_{n}}\left(\frac{t}{\left(1-2 t^{h}\right)^{2}}, s, s\right)\right| d s\right] d \sigma+C t^{\frac{-1}{2(1+\alpha)}+\frac{\delta^{\prime}}{4}}, \quad t \rightarrow 0,
\end{aligned}
$$

where $\widetilde{P}_{n}=P_{n}-p_{0}^{1}$ and $P_{n}$ is the 1-dimensional Green function on the interval $l_{n}$.

Absolutely similarly one can get a lower bound estimate for the integral in the left-hand side of (44). We start with formula (38), add two small intervals of the length $\delta_{n}=\varepsilon^{(1+\alpha) \delta}\left|l_{n}\right|$ to the ends of the segments $l_{n}$ and denote the extended segments by $l_{n}^{+}$. Let $p_{n}^{+}$be the Green function for the layer bounded by the planes through the ends of $l_{n}^{+}$which are orthogonal to $l_{n}^{+}$. Let $\widetilde{p_{n}^{+}}=p_{n}^{+}-p_{0}$. Let $R_{n}^{\varepsilon}$ be the domain bounded by $\Gamma_{n}, \Gamma_{n+1}$ and by the lateral surface of the circle cylinder of radius $\varepsilon$ with axes $l_{n}^{+}$. Similar to (41) one can compare $p$ and $p_{n}^{+}$in $R_{n}^{\varepsilon}$ and show that

$$
p \leqq p_{n}^{+}+C t \quad \text { when } x \in R_{n}^{\varepsilon}, y \in l_{n}, t \leqq t_{0},
$$

and therefore

$$
\widetilde{p} \geqq \widetilde{p_{n}^{+}}-C t \quad \text { when } x \in R_{n}^{\varepsilon}, y \in l_{n}, t \leqq t_{0} .
$$

We can replace $\widetilde{p_{n}^{+}}(t, x, x)$ by $(4 \pi t)^{(1-d) / 2} \widetilde{P_{n}^{+}}(t, s, s)$, where $x=x(s, u), \widetilde{P_{n}^{+}}=$ $P_{n}^{+}-p_{0}^{1}$ and $P_{n}^{+}$is the one dimensional Green function for the segment $l_{n}^{+}$. If we substitute the result in (38) and put $t=t_{0}$, we will get the following estimate:

$$
(4 \pi t)^{(d-1) / 2} \int_{\Omega}|\widetilde{p}(t, x, x)| d x \geqq \sum_{n_{1} \geqq n \geqq n_{2}} \int_{\Gamma^{\prime}}\left[\int_{l_{n}}\left|\widetilde{P_{n}^{+}}(t, s, s)\right| d s\right] d \sigma-C t^{\frac{-1}{2(1+\alpha)}+\frac{\delta^{\prime}}{4}}, \quad t \rightarrow 0 .
$$

The sum of the lengths $\left|l_{n}\right|$ does not exceed the value (34). The sum of the lengths of added intervals is equal to the $\varepsilon^{(1+\alpha) \delta}$-part of the first sum and does not exceed $C \varepsilon^{\frac{\alpha}{1+\alpha}+\delta} \leqq C t^{\frac{\alpha}{2(1+\alpha)}+\frac{\delta}{4}}$ if $v$ is small enough (see also the proof of (31)). Together with the estimate $\left|\widetilde{P_{n}^{+}}(t, s, s)\right| \leqq(4 \pi t)^{-1 / 2}$ it allows us to replace $l_{n}$ in (45) 
by $l_{n}^{+}$because the error due to this change is not greater than the last term in the right-hand side of (45). After this we can use (43) and similar to (44) we get

$$
\begin{aligned}
& (4 \pi t)^{\frac{d-1}{2}} \int_{\Omega}|\widetilde{p}(t, x, x)| d x \\
& \geqq \sum_{n_{1} \geqq n \geqq n_{2}} \int_{\Gamma^{\prime}}\left[\int_{l_{n}}\left|\widetilde{P}_{n}\left(\frac{t}{\left(1+2 t^{h}\right)^{2}}, s, s\right)\right| d s\right] d \sigma-C t^{\frac{-1}{2(1+\alpha)}+\frac{\delta^{\prime}}{4}}, \quad t \rightarrow 0 .
\end{aligned}
$$

These two main estimates (44) and (46) are valid for any dimension $d \geqq 1$, and in particular for $d=1$. Let us recall that we use $s=|x-\sigma|$ as coordinates on the segment $l^{\prime}(\sigma)$ of the ray $l(\sigma)$, and that $P\left(t, s, s^{\prime}\right)$ is the 1-dimensional Green function on the set $\left\{l_{n}\right\}$ of intervals $l_{n} \subset l^{\prime}(\sigma)$ with Dirichlet boundary conditions at points $x_{n}=l^{\prime}(\sigma) \cap \Gamma_{n}, n=1,2,3 \ldots$ Let $\widetilde{P}=P-p_{0}^{1}$. Then estimates (44), (46) remain valid if we replace their left-hand sides by $\int_{l^{\prime}(\sigma)} \widetilde{P}(t, s, s) d s$ (in fact if we were interested in estimates only for $d=1$ we could get them easier). For a given small $\tau>0$ let us find $t=f(\tau)$ such that $\frac{t}{\left(1-2 t^{h}\right)^{2}}=\frac{\tau}{\left(1+2 \tau^{h}\right)^{2}}$. Then the 1-dimensional versions of (44), (46) can be written in the following form:

$$
\begin{aligned}
& \int_{l^{\prime}(\sigma)} \widetilde{P}(f(\tau), s, s) d s \leqq \sum_{n_{1} \geqq n \geqq n_{2} l_{n}} \int\left|\widetilde{P}_{n}\left(\frac{\tau}{\left(1+2 \tau^{h}\right)^{2}}, s, s\right)\right| d s+C \tau^{\frac{-1}{2(1+\alpha)}+\frac{\delta^{\prime}}{4}}, \quad \tau \rightarrow 0, \\
& \int_{l^{\prime}(\sigma)} \widetilde{P}\left(f^{-1}(\tau), s, s\right) d s \geqq \sum_{n_{1} \geqq n \geqq n_{2}} \int_{l_{n}}\left|\widetilde{P}_{n}\left(\frac{\tau}{\left(1-2 \tau^{h}\right)^{2}}, s, s\right)\right| d s-C \tau^{\frac{-1}{2(1+\alpha)}+\frac{\delta^{\prime}}{4}}, \quad \tau \rightarrow 0
\end{aligned}
$$

respectively. Here $f^{-1}$ is the inverse function.

Combining (44) with (48) and (46) with (47) we obtain that

$$
\begin{aligned}
& \int_{\Gamma^{\prime} l^{\prime}(\sigma)} \tilde{P}(f(t), s, s) d s d \sigma-C t^{\frac{-1}{2(1+\alpha)}+\frac{\delta^{\prime}}{4}} \leqq(4 \pi t)^{(d-1) / 2} \int_{\Omega}|\widetilde{p}(t, x, x)| d x \\
& \quad \leqq \int_{\Gamma^{\prime}} \int_{l^{\prime}(\sigma)} \widetilde{P}\left(f^{-1}(t), s, s\right) d s d \sigma+C t^{\frac{-1}{2(1+\alpha)}+\frac{\delta^{\prime}}{4}}, \quad t \rightarrow 0 .
\end{aligned}
$$

From the relations $f(t) \sim t$ and $f^{-1}(t) \sim t$ as $t \rightarrow 0$ and from Lemma 4 it follows that integrals over $l^{\prime}(\sigma)$ in (49) have order $O\left(t^{\frac{-1}{2(1+\alpha)}}\right)$ as $t \rightarrow 0$. Together with the fact that $\operatorname{mes}\left(\Gamma \backslash \Gamma^{\prime}\right) \leqq C d_{\varepsilon} \leqq C t^{\sigma}$, where $\sigma=\left(\frac{\alpha}{1+\alpha}-\alpha \delta\right)\left(\frac{1}{2}-v\right)>0$ it allows us to replace $\Gamma^{\prime}$ by $\Gamma$ in (49). After this formula (49) together with Lemma 4 give the assertion of Theorem 5 .

Theorem 5 is proved.

\section{Bubble Type Domains}

Definition 6. Domain $\Omega \subset \Re^{3}$ is called a bubble type domain if

$$
\Omega=\Omega_{0} \backslash\left(\bigcup_{j=1}^{\infty} B_{j}\right),
$$

where $\Omega_{0}$ is a bounded domain with the smooth boundary $\partial \Omega_{0}$ and $\left\{B_{j}\right\}$ is a set of nonintersecting balls $B_{j} \in \Omega_{0}$ of radii $r_{j}$ with the centers at $x_{j} \in \Omega_{0}$. 
In this article we assume additionally that $\sum_{1}^{\infty} r_{j}<\infty$. In our next publication we will consider a more general case.

Let $\mathbf{R}_{\lambda}$ be the resolvent of the Dirichlet problem for the Laplace operator in $\Omega$, and $K_{\lambda}(x, y)$ be the Shwartz kernel of the operator $\mathbf{R}_{\lambda}$, i.e. $K_{\lambda}$ is the Green function of the Dirichlet problem for the operator $\Delta-\lambda$. We would like to study the integral

$$
\int_{\Omega} K_{\lambda}(x, x) d x, \quad \lambda \rightarrow \infty
$$

which corresponds to $-\varsigma^{-}(\lambda)$ (see (3)) with $z=1$. However, the kernel $K_{\lambda}(x, y)$ has a singularity as $x \rightarrow y$. That is why we have to consider the iteration $\left(\mathbf{R}_{\lambda}\right)^{2}$ of the operator $\mathbf{R}_{\lambda}$ and its kernel $G_{\lambda}(x, y)$ which is the solution of the problem

$$
\begin{gathered}
(\Delta-\lambda)^{2} G_{\lambda}=\delta(x-y), \quad x, y \in \Omega \subset \Re^{3}, \\
G_{\lambda}=(\Delta-\lambda) G_{\lambda}=0 \quad \text { on } \partial \Omega .
\end{gathered}
$$

The purpose of this section is to show that Weyl's asymptotics with two main terms is valid for the integral

$$
\int_{\Omega} G_{\lambda}(x, x) d x, \quad \lambda \rightarrow \infty,
$$

which corresponds to $\varsigma^{-}(\lambda)$ with $z=2$. It indicates that the spectral dimension of the boundary $\partial \Omega$ is 2 . We also show that at the same time the Minkowski dimension of $\partial \Omega$ can be an arbitrary number in the interval $(2,3)$.

Now we formulate the main result of the section

Theorem 7. Let $\Omega$ be a domain of bubble type and $\sum r_{i}<\infty$. Then the following formula is valid for the Green function $G_{\lambda}$ of problem (50):

$$
\int_{\Omega} G_{\lambda}(x, x) d x=\frac{\operatorname{vol} \Omega}{8 \pi \sqrt{\lambda}}-\frac{\operatorname{mes} \partial \Omega_{0}+4 \pi \sum r_{i}^{2}}{16 \pi \lambda}+o(\lambda), \quad \lambda \rightarrow \infty .
$$

We need several lemmas in order to prove the theorem. The following lemma is an obvious consequence of the maximum principle for the Laplace operator.

Lemma 8 (Maximum Principle). Let $Q$ be a bounded domain and

$$
\begin{gathered}
(\Delta-\lambda)^{2} u=0 \quad \text { in } Q, \\
u \leqq 0, \quad(\Delta-\lambda) u \geqq 0 \quad \text { on } \partial Q .
\end{gathered}
$$

Then $u \leqq 0$ in $Q$. This assertion is also valid if $Q$ is an exterior domain and $u \rightarrow 0$ as $|x| \rightarrow \infty$.

Proof. In order to prove Lemma 8 in the case of bounded $Q$ one can apply the maximum principle for the operator $\Delta-\lambda$ successively to the functions $(-\Delta+\lambda) u$ and $u$. If $Q$ is an exterior domain it must be noted first that all derivatives of $u$ tend to zero at infinity if $u$ satisfies the equation $(\Delta-\lambda)^{2} u=0$ in $Q$ and $u \rightarrow 0$ as $|x| \rightarrow \infty$. The lemma is proved.

The next four lemmas are devoted to studying the Green function $G_{\lambda}$ of the operator $(\Delta-\lambda)^{2}$ in the exterior of the ball $B(R)$ of the radius $R$ with the center at the origin. We will denote this Green function by $T_{\lambda}=T_{\lambda}(x, y ; R)$. Our purpose is to find an asymptotic behavior of $T_{\lambda}(x, y ; R)$ when $x=y, \lambda \rightarrow \infty, R \rightarrow 0$. First of all let us make two remarks. 
Remark 3. The definition of the Green function $G_{\lambda}$ for an exterior domain $\left(\Re^{3} \backslash B(R)\right.$, for example) includes relations (50) and also a decay of $G_{\lambda}$ at infinity:

$$
\lim _{|x| \rightarrow \infty} G_{\lambda}=0
$$

Remark 4. Function $E=\frac{\exp (-\sqrt{\lambda}|x-y|)}{8 \pi \sqrt{\lambda}}$ is a fundamental solution of the operator, i.e.

$$
(\Delta-\lambda)^{2} E=\delta(x-y) \text {. }
$$

In particular,

$$
(\Delta-\lambda)^{2} \frac{\exp (-\sqrt{\lambda}|x-y|)}{\sqrt{\lambda}}=0 \text { as } x \neq y .
$$

Lemma 9. Let $(\Delta-\lambda)^{2} u=0$ outside of the ball $B(R)$, and

$$
|u| \leqq \alpha, \quad|(\Delta-\lambda) u| \leqq 2 \beta \quad \text { when }|x|=R ; \quad u \rightarrow 0 \quad \text { as }|x| \rightarrow \infty .
$$

Then

$$
|u| \leqq\left(\frac{\alpha R}{|x|}+\frac{\beta R}{\sqrt{\lambda}}\right) e^{-\sqrt{\lambda}(|x|-R)} \quad \text { for }|x| \geqq R .
$$

Proof. Let us denote the right-hand side in (55) by $v$. Due to (54), we have

$$
\begin{gathered}
(\Delta-\lambda)^{2} v=0, \quad x \neq 0, \\
\left.v\right|_{|x|=R}=\alpha+\frac{\beta R}{\sqrt{\lambda}} \geqq \alpha,\left.\quad(\Delta-\lambda) v\right|_{|x|=R}=-2 \beta .
\end{gathered}
$$

Hence, $\pm u-v$ satisfy assumptions of Lemma 8 with $Q=\Re^{3} \backslash B(R)$, and therefore $\pm u-v \leqq 0$ in $\Re^{3} \backslash B(R)$. Lemma 9 is proved.

If $|y| \geqq R$ we will denote by $y^{*}$ the point conjugated to $y$ with respect to the sphere $|x|=R$, i.e. $y^{*}$ belongs to the segment $[0, y]$ and $\left|y^{*}\right| \cdot|y|=R^{2}$.

Lemma 10. For any $R, \lambda>0,|x| \geqq R,|y|>R$, the following formula is valid:

$$
T_{\lambda}(x, y ; R)=\frac{1}{8 \pi \sqrt{\lambda}} e^{-\sqrt{\lambda}|x-y|}-\frac{R}{8 \pi \sqrt{\lambda}|y|} e^{-\sqrt{\lambda}\left|x-y^{*}\right|}+g,
$$

where

$$
|g| \leqq \frac{R}{8 \pi}(|y|-R) e^{-\sqrt{\lambda}(|x|-R)}\left(\frac{1}{\sqrt{\lambda}|x||y|}+\frac{2}{|x|}+\frac{1}{|y|}\right) .
$$

Proof. Recall that

$$
|x-y|=\left|x-y^{*}\right| \frac{|y|}{R} \quad \text { if }|x|=R .
$$


From here, (56) and the first boundary condition in (50) it follows that on $\partial B(R)$

$$
\begin{aligned}
g & =\frac{1}{8 \pi \sqrt{\lambda}}\left[\frac{R}{|y|} e^{-\sqrt{\lambda}\left|x-y^{*}\right|}-e^{-\sqrt{\lambda}|x-y|}\right] \\
& =\frac{1}{8 \pi \sqrt{\lambda}}\left[\frac{R}{|y|} e^{-\sqrt{\lambda}\left|x-y^{*}\right|}-e^{-\sqrt{\lambda}\left|x-y^{*}\right| \frac{|y|}{R}}\right] \\
& =\frac{1}{8 \pi \sqrt{\lambda}} e^{-\sqrt{\lambda}\left|x-y^{*}\right|}\left[\frac{R}{|y|}-1+1-e^{-\sqrt{\lambda}\left|x-y^{*}\right|\left(\frac{|y|}{R}-1\right)}\right] .
\end{aligned}
$$

Since $|y|>R$, we have

$$
\left|1-e^{-\sqrt{\lambda}\left|x-y^{*}\right|\left(\frac{|y|}{R}-1\right)}\right| \leqq \sqrt{\lambda}\left|x-y^{*}\right|\left(\frac{|y|}{R}-1\right) \leqq 2 \sqrt{\lambda}(|y|-R) .
$$

In order to get the last inequality we used the facts that $\left|y^{*}\right|<R$ and $\left|x-y^{*}\right| \leqq$ $|x|+\left|y^{*}\right| \leqq 2 R$ for $|x|=R$. From (59) and (60) we have

$$
\begin{aligned}
|g| & \leqq \frac{1}{8 \pi \sqrt{\lambda}}\left[1-\frac{R}{|y|}+2 \sqrt{\lambda}(|y|-R)\right] \\
& =\frac{|y|-R}{8 \pi}\left(\frac{1}{\sqrt{\lambda}|y|}+2\right), \quad|x|=R .
\end{aligned}
$$

Formula (56), the second boundary condition in (50) and (58) together lead to the following relations when $x \in \partial B(R)$ :

$$
\begin{aligned}
(\Delta-\lambda) g & =(\Delta-\lambda)\left[\frac{R}{8 \pi \sqrt{\lambda}|y|} e^{-\sqrt{\lambda}\left|x-y^{*}\right|}-\frac{1}{8 \pi \sqrt{\lambda}} e^{-\sqrt{\lambda}|x-y|}\right] \\
& =-\frac{R}{4 \pi|y|\left|x-y^{*}\right|} e^{-\sqrt{\lambda}\left|x-y^{*}\right|}+\frac{1}{4 \pi|x-y|} e^{-\sqrt{\lambda}|x-y|} \\
& =\frac{R}{4 \pi|y|\left|x-y^{*}\right|}\left(e^{-\sqrt{\lambda}\left|x-y^{*}\right| \frac{|y|}{R}}-e^{\sqrt{\lambda}\left|x-y^{*}\right|}\right) \\
& =\frac{R}{4 \pi|y|\left|x-y^{*}\right|} e^{-\sqrt{\lambda}\left|x-y^{*}\right|}\left[e^{-\sqrt{\lambda}\left|x-y^{*}\right|\left(\frac{|y|}{R}-1\right)}-1\right] .
\end{aligned}
$$

From here and the first of inequalities (60) we get that

$$
|(\Delta-\lambda) g| \leqq \frac{\sqrt{\lambda}(|y|-R)}{4 \pi|y|}, \quad|x|=R .
$$

From (56) and Remarks 3,4 it follows that $(\Delta-\lambda)^{2} g=0$ in $\Re^{3} \backslash B(R)$ and $g \rightarrow 0$ as $|x| \rightarrow \infty$. Together with (61),(62) and (55) it gives (57). Lemma 10 is proved. Lemma 11. For any $R, \lambda>0$ and $|x| \geqq R$, $\left|T_{\lambda}(x, x ; R)-\frac{1}{8 \pi \lambda}\right| \leqq \frac{R}{8 \pi \sqrt{\lambda}|x|} e^{-\sqrt{\lambda}(|x|-R)}+\frac{R}{8 \pi}(|x|-R) e^{-\sqrt{\lambda}(|x|-R)}\left(\frac{1}{\sqrt{\lambda}|x|^{2}}+\frac{3}{|x|}\right)$. 
Proof. This estimate follows immediately from Lemma 10 and the inequality $\left|x-x^{*}\right| \geqq|x|-\left|x^{*}\right| \geqq|x|-R$.

Now we can prove the final assertion on the Green function for the exterior of $B(R)$.

Lemma 12. For any $R, \lambda>0$,

$$
\int_{|x|>R}\left|T_{\lambda}(x, x ; R)-\frac{1}{8 \pi \sqrt{\lambda}}\right| d x \leqq \frac{2 R^{2}}{\lambda}+\frac{4 R}{\lambda^{3 / 2}} .
$$

Proof. Let us integrate (63) over the exterior of the ball $B(R)$. We have

$$
\frac{1}{8 \pi \sqrt{\lambda}} \int_{|x|>R} \frac{1}{|x|} e^{-\sqrt{\lambda}(|x|-R)} d x=\frac{1}{2 \sqrt{\lambda}} \int_{R}^{\infty} r e^{\sqrt{\lambda}(r-R)} d r=\frac{R}{2 \lambda}+\frac{1}{2 \lambda^{3 / 2}} .
$$

Similarly

$$
\begin{aligned}
& \frac{1}{8 \pi \sqrt{\lambda}} \int_{|x|>R} \frac{|x|-R}{|x|^{2}} e^{-\sqrt{\lambda}(|x|-R)} d x=\frac{1}{2 \lambda^{3 / 2}}, \\
& \frac{1}{8 \pi} \int_{|x|>R} \frac{|x|-R}{|x|} e^{-\sqrt{\lambda}(|x|-R)} d x=\frac{R}{2 \lambda}+\frac{1}{\lambda^{3 / 2}} .
\end{aligned}
$$

It leads to (64). Lemma 12 is proved.

We will need three more lemmas in order to prove Theorem 7.

Let $T_{i}$ be the Green function (see (50) and Remark 3) in the exterior of the ball $B_{i}$, i.e. $T_{i}=T_{\lambda}\left(x-x_{i}, y-x_{i} ; r_{i}\right)$. Let $G_{\lambda}^{N}=G_{\lambda}^{N}(x, y)$ be the Green function for domain $\Omega_{N}=\Omega_{0} \backslash\left(U_{i \leqq N} B_{i}\right)$. Let

$$
G_{\lambda}=E-\widetilde{G}_{\lambda}, \quad G_{\lambda}^{N}=E-\widetilde{G}_{\lambda}^{N}, \quad T_{i}=E-\widetilde{T}_{i},
$$

where $E$ is the fundamental solution of the operator $(\Delta-\lambda)^{2}$ (see Remark 4). Then $\widetilde{G}_{\lambda}, \widetilde{G}_{\lambda}^{N}, \widetilde{T}_{i}$ are the solutions of the homogeneous equations in domains $\Omega, \Omega_{N}, \Re^{3} \backslash B_{i}$ respectively with corresponding inhomogeneous boundary conditions. In particular,

$$
(\Delta-\lambda)^{2} \widetilde{G}_{\lambda}=0, \quad x \in \Omega ; \quad \widetilde{G}_{\lambda}=E, \quad(\Delta-\lambda) \widetilde{G}_{\lambda}=(\Delta-\lambda) E, \quad x \in \partial \Omega .
$$

Lemma 13. The following inequalities hold:

$$
0<\widetilde{G}_{\lambda}, \widetilde{G}_{\lambda}^{N}, \widetilde{T}_{i} \leqq E .
$$

Proof. These inequalities for all three functions can be proved in the same way. Let us prove them for $\widetilde{G}_{\lambda}$, for instance. Let $u$ be a solution of the problem.

$$
(\Delta-\lambda) u=\delta(x-y) \quad \text { in } \Omega, \quad u=0 \quad \text { on } \partial \Omega .
$$

From the maximum principle for the operator $\Delta-\lambda$, it follows that $u \leqq 0$ in $\Omega$. Thus, $G_{\lambda}$ is the solution of the problem

$$
(\Delta-\lambda) G_{\lambda}=u \leqq 0 \quad \text { in } \Omega, \quad G_{\lambda}=0 \quad \text { on } \partial \Omega,
$$

and the minimum principle for solutions of (66) leads to the inequality $G_{\lambda} \geqq 0$. Hence $\widetilde{G}_{\lambda} \leqq E$. Inequality $\widetilde{G}_{\lambda}>0$ follows from (65) and Lemma 8 since $E>0$ and $(\Delta-\lambda) E=-\frac{e^{-\sqrt{\lambda}|x-y|}}{4 \pi|x-y|}<0$. Lemma 13 is proved. 
Lemma 14. The following inequalities hold:

$$
0 \leqq \widetilde{G}_{\lambda}-\widetilde{G}_{\lambda}^{N} \leqq \sum_{i>N} \widetilde{T}_{i}, \quad x, y \in \Omega .
$$

Proof. Since $\widetilde{G}_{\lambda}=\widetilde{G}_{\lambda}^{N}=E$ on $\partial \Omega_{N}$ and $\widetilde{T}_{i}>0$ (due to Lemma 13), we have

$$
0=\widetilde{G}_{\lambda}-\widetilde{G}_{\lambda}^{N}<\sum_{i>N} \widetilde{T}_{i}, \quad x \in \partial \Omega_{N}
$$

If $x \in \partial B_{j}, j>N$, then $\widetilde{G}_{\lambda}=E$ and $0<\widetilde{G}_{\lambda}^{N} \leqq E$ (due to Lemma 13). Hence

$$
0 \leqq \widetilde{G}_{\lambda}-\widetilde{G}_{\lambda}^{N}<E, \quad x \in \partial B_{j}, \quad j>N .
$$

On the other hand $\widetilde{T}_{j}=E$ on $\partial B_{j}$ and $\widetilde{T}_{i}>0$ for any $i$, and therefore $\sum_{i>N} \widetilde{T}_{i}>E$ on $\partial B_{j}$ if $j>N$. Together with (68) and (67) it leads to the inequalities

$$
0 \leqq \widetilde{G}_{\lambda}-\widetilde{G}_{\lambda}^{N}<\sum_{i>N} \widetilde{T}_{i}, \quad x \in \partial \Omega_{N} .
$$

Similarly one can check that

$$
0 \geqq(\Delta-\lambda)\left(\widetilde{G}_{\lambda}-\widetilde{G}_{\lambda}^{N}\right) \geqq \sum_{i>N}(\Delta-\lambda) \widetilde{T}_{i}, \quad x \in \partial \Omega .
$$

The assertion of Lemma 14 follows immediately from (69), (70) and Lemma 8. Lemma 15. Let $Q$ be a bounded domain with the smooth boundary, and $K_{\lambda}=$ $K_{\lambda}(x, y)$ be the Green function in $Q$ :

$$
(\Delta-\lambda)^{2} K_{\lambda}=\delta(x-y) \quad \text { in } Q ; \quad K_{\lambda}=(\Delta-\lambda) K_{\lambda}=0 \quad \text { when } x \in \partial Q .
$$

Then the following estimate is valid for the function $\widetilde{K}_{\lambda}=\frac{\exp (-\sqrt{\lambda}|x-y|)}{8 \pi \sqrt{\lambda}}-K_{\lambda}$ :

$$
\left|\widetilde{K}_{\lambda}(x, x)\right| \leqq \frac{3 e^{-\sqrt{\lambda} \rho(x, \partial Q)}}{4 \pi \sqrt{\lambda}} .
$$

Proof. Let $Q_{y}$ be a cube with the center at $y \in Q$ and the edges equal to $d=$ $\rho(y, \partial Q)$. Then $Q_{y} \in Q$. We denote by $P_{\lambda}=P_{\lambda}(x, y)$ the Green function of problem (71) in the cube $Q_{y}$ (with $\delta$-function at the center of the cube). Since function $u=(\Delta-\lambda) K_{\lambda}$ satisfies relations $(\Delta-\lambda) u=\delta(x-y)$ in $Q$ and $u=0$ on $\partial Q$, it follows from the maximum principle for the operator $(\Delta-\lambda)$ that $(\Delta-\lambda) K_{\lambda} \geqq 0$ on $Q$. In particular, $(\Delta-\lambda) K_{\lambda} \geqq 0$ on $\partial Q_{y}$. From the assertion of Lemma 13 with $\Omega=Q$ it follows that $K_{\lambda} \geqq 0$ on $Q$. Thus, $K_{\lambda} \geqq 0$ on $\partial Q_{y}$. Applying Lemma 8 to $K_{\lambda}-P_{\lambda}$ in $Q_{y}$ we get that

$$
K_{\lambda}(x, y) \geqq P_{\lambda}(x, y), \quad x \in Q_{y} .
$$

Thus, if $\widetilde{P}_{\lambda}=\frac{\exp (-\sqrt{\lambda}|x-y|)}{8 \pi \sqrt{\lambda}}-P_{\lambda}$ then

$$
\widetilde{K}_{\lambda}(x, y) \leqq \widetilde{P}_{\lambda}(x, y), \quad x \in Q_{y} .
$$


Let us reflect point $y$ with respect to faces of the cube $Q_{\lambda}$ and denote reflected points by $y_{j}, 1 \leqq j \leqq 6$. One can easily check that

$$
\sum_{j=1}^{j=6} \frac{e^{\left(-\sqrt{\lambda}\left|x-y_{j}\right|\right)}}{8 \pi \sqrt{\lambda}} \geqq \widetilde{P}_{\lambda}(x, y), \quad(\Delta-\lambda) \sum_{j=1}^{j=6} \frac{e^{\left(-\sqrt{\lambda}\left|x-y_{j}\right|\right)}}{8 \pi \sqrt{\lambda}} \leqq(\Delta-\lambda) \widetilde{P}_{\lambda}(x, y)
$$

when $x \in \partial Q_{y}$. Together with Lemma 8 it gives the first inequality for any $x \in Q_{y}$. From here and (72) we get that

$$
\widetilde{K}_{\lambda}(x, y) \leqq \sum_{j=1}^{j=6} \frac{e^{\left(-\sqrt{\lambda}\left|x-y_{j}\right|\right)}}{8 \pi \sqrt{\lambda}}, \quad x \in Q_{y} .
$$

Similarly to Lemma 13 we have that $\widetilde{K}_{\lambda}(x, y) \geqq 0$. Assertion of Lemma 15 follows from here and (73) if we put $x=y$.

Lemma 15 is proved.

Proof of Theorem 7. We fix an arbitrary $\varepsilon>0$ and choose $N=N(\varepsilon)$ such that

$$
\sum_{i>N(\varepsilon)}\left(2 r_{i}^{2}+4 r_{i}\right)<\frac{\varepsilon}{4}, \quad \sum_{i>N(\varepsilon)} r_{i}^{2}<\frac{\varepsilon}{4} .
$$

From Lemmas 12, 14 and formula (74) we get that

$$
0 \leqq \int_{\Omega}\left(G_{\lambda}^{N}(x, x)-G_{\lambda}(x, x)\right) d x \leqq \frac{\varepsilon}{4 \lambda} .
$$

Since $\Omega_{N}$ is a domain with a smooth boundary then from the Minakshasandaram expansion it follows that

$$
\int_{\Omega_{N}} G_{\lambda}^{N}(x, x) d x=\frac{\operatorname{vol} \Omega_{N}}{8 \pi \sqrt{\lambda}}-\frac{\operatorname{mes}\left(\partial \Omega_{0}\right)+4 \pi \sum_{i=1}^{N} r_{i}^{2}}{16 \pi \lambda}+o\left(\frac{1}{\lambda^{3 / 2}}\right), \quad \lambda \rightarrow \infty .
$$

We fix $\lambda_{1}=\lambda_{1}(\varepsilon)$ such that the remainder is less than $\varepsilon / 4 \lambda$ when $\lambda \geqq \lambda_{1}$. Then taking into account the second of the estimates (74) we can rewrite (76) in the form

$$
\left|\int_{\Omega_{N}} G_{\lambda}^{N}(x, x) d x-\frac{\operatorname{vol} \Omega_{N}}{8 \pi \sqrt{\lambda}}+\frac{\operatorname{mes}\left(\partial \Omega_{0}\right)+4 \pi \sum_{i=1}^{\infty} r_{i}^{2}}{16 \pi \lambda}\right| \leqq \frac{\varepsilon}{2 \lambda}, \quad \lambda>\lambda_{1} .
$$

Now we are going to show that

$$
\left|\int_{j>N} G_{\lambda}^{N}(x, x) d x-\frac{\operatorname{vol}\left(\Omega \backslash \Omega_{N}\right)}{8 \pi \sqrt{\lambda}}\right| \leqq \frac{\varepsilon}{4 \lambda}, \quad \lambda>0 .
$$

If $r$ is the distance between $x \in B_{j}$ and the center of the ball $B_{j} \subset \Omega$, then $\rho(x, \partial \Omega) \geqq r_{j}-r$, where $r_{j}$ is the radius of $B_{j}$. From here and Lemma 15 it follows 
that

$$
\begin{aligned}
\left|\int_{B_{j}} \widetilde{G}_{\lambda}^{N}(x, x) d x\right| & \leqq \frac{3}{4 \pi \sqrt{\lambda}} \int_{B_{j}} e^{-\sqrt{\lambda} \rho} d x \leqq \frac{3}{4 \pi \sqrt{\lambda}} \int_{0}^{r_{j}} e^{-\sqrt{\lambda}\left(r_{j}-r\right) r^{2}} d r \\
& \leqq \frac{3 r_{j}^{2}}{4 \pi \lambda}<\frac{r_{j}^{2}}{\lambda}, \quad j>N .
\end{aligned}
$$

Together with the first of estimates (74) it leads to the inequality

$$
\left|\bigcup_{j>N} \widetilde{G}_{\lambda}^{N}(x, x) d x\right|<\frac{\varepsilon}{4 \lambda}, \quad \lambda>0,
$$

which in its turn gives (78) because $G_{\lambda}^{N}(x, x)=\frac{1}{8 \pi \sqrt{\lambda}}-\widetilde{G}_{\lambda}^{N}(x, x)$. From (75), (77) and (78) we get that

$$
\left|\int_{\Omega} G_{\lambda}(x, x) d x-\frac{\operatorname{vol} \Omega}{8 \pi \sqrt{\lambda}}+\frac{\operatorname{mes}\left(\partial \Omega_{0}\right)+4 \pi \sum_{i=1}^{\infty} r_{i}^{2}}{16 \pi \lambda}\right| \leqq \frac{\varepsilon}{\lambda}, \quad \lambda>\lambda_{1}(\varepsilon) .
$$

Since $\varepsilon$ is arbitrary, it completes the proof of Theorem 7.

The last part of the section contains a proof of Minkowski measurability for two classes of bubble type domains. We will return to these classes of domains in our next publication. Here our objective is only to show that under assumptions of Theorem 7 the Minkowski dimension can be an arbitrary number in the interval $2<m<3$ at the same time as the spectral dimension is 2 (due to Theorem 7).

3.1. $(\alpha, \beta, \gamma)$-model. Now we describe a class of bubble type domains in $\Re^{3}$ which depends on three parameters $\alpha, \beta$ and $\gamma$ with $\beta>\alpha>1, \alpha-1>\gamma>0$. Suppose that domain $\Omega_{0}$ contains the unit cube $Q$, and the lower face of cube $Q$ belongs to the boundary $\partial \Omega_{0}$ of the domain (see Fig. 5 ). Let's consider a partition of $Q$ by layers $L_{n}, n=1,2,3 \ldots$, of the size $\Delta_{n}, \sum_{n=1}^{\infty} \Delta_{n}=1$. We assume that the layers are united into groups $\Gamma_{i}$, each group $\Gamma_{i}$ contains $k_{i}$ layers with the same widths $l_{i}$ :

$$
\Delta_{1}=\cdots=\Delta_{k_{1}}=l_{1}, \quad \Delta_{k_{1}+1}=\cdots=\Delta_{k_{1}+k_{2}}=l_{2}, \quad \ldots
$$

Of course $\sum k_{i} l_{i}=1$.

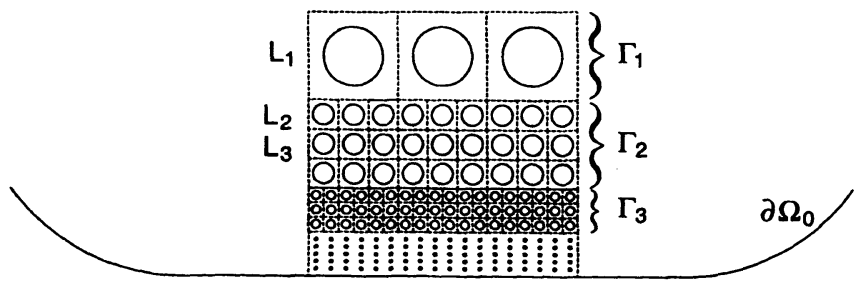

Fig. 5 
We assume that $N_{i}=1 / l_{i}$ are integers, and the following two relations are fulfilled:

$$
N_{i} \sim c_{0} i^{\alpha}, \quad k_{i} \sim c_{1} i^{\gamma} \quad \text { as } i \rightarrow \infty
$$

We represent each layer from $\Gamma_{i}$ as the union of the equal subcubes $Q_{i, j} \subset L_{i}$, $j=1,2, \ldots, N_{i}^{2}$. Let $B_{i, j}$ be a ball of radius $r_{i, j}=r_{i}$ centered at the central point $x_{i, j}$ of $Q_{i, j}$, and $r_{i} \sim c_{2} i^{-\beta}$ as $i \rightarrow \infty$. Of course, $r_{i}<l_{i}$ if $i$ is big enough $(\beta>\alpha)$, but we assume that this inequality is fulfilled for all values of $i$. It means that balls $B_{i, j}$ are not overlapping.

Definition 16. The $(\alpha, \beta, \gamma)$-model of a bubble type domain $\Omega$ considered below has the form

$$
\Omega=\Omega_{0} \backslash \bigcup_{i, j} B_{i, j} .
$$

Remark 5. Let's note that

$$
\sum_{i, j} r_{i, j}=\sum_{i} k_{i} N_{i}^{2} r_{i}<\infty \quad \text { if } \beta>2 \alpha+\gamma+1
$$

because $k_{i} \sim c_{1} i^{\gamma}, N_{i} \sim c_{0} i^{\alpha}, r_{i} \sim c_{2} i^{-\beta}$ as $i \rightarrow \infty$. Thus, Theorem 7 can be applied for domain (18) if $\beta>2 \alpha+\gamma+1$. It means that in this case $(\beta>2 \alpha+\gamma+1)$ the second term of the spectral asymptotics does not depend on constants $\alpha, \beta, \gamma$, and it has the same order as for domains with smooth boundaries. On the other hand we will show below that $\partial \Omega$ is Minkowski measurable with $m(\partial \Omega)=2+\frac{\gamma+1}{\alpha}$. Thus, the Minkowski dimension $m(\partial \Omega)$ can be an arbitrary number between 2 and 3 $(\alpha-1>\gamma>0)$, and the MWB conjecture is not valid.

Remark 6. One can consider bubble type domains in space $\Re^{d}$ with any $d>2$. In this case, a theorem similar to Theorem 7 is valid with the assumption that $\sum_{i} r_{i}^{d-2}<\infty$. The definition of $(\alpha, \beta, \gamma)$-model in $d$-dimensional space is the same as for $d=3$, and the analogue of Theorem 7 can be applied to this model if $\beta>\frac{\alpha(d-1)+\gamma+1}{d-2}$. It means that under this assumption the second term of the spectral asymptotics is the same as for domains with smooth boundaries. On the other hand, in the $d$-dimensional case $m(\partial \Omega)=d-1+\frac{\gamma+1}{\alpha}$, and it is an arbitrary number between $d-1$ and $d$.

For simplicity we will consider below only $d=3$, although there are no difficulties to take an arbitrary $d$. In order to formulate the next theorem we need function $F=F(r)$, which is the volume of the part of a unit cube covered by the set of 8 balls of the radii $r$ centered at vertices of the cube. Of course $F(r)=\frac{4}{3} \pi r^{3}$ if $r \leqq 1 / 2$, and $F(r)=1$ if $r \geqq \sqrt{3} / 2$.

Theorem 17. If $\beta>\alpha>1, \alpha-1>\gamma>0$, then the boundary of the domain $\Omega=$ $\Omega_{0} \backslash \bigcup_{i, j} B_{i, j} \in \Re^{3}$ is Minkowski measurable with Minkowski dimension and Minkowski content equal respectively to

$$
m(\partial \Omega)=2+\frac{\gamma+1}{\alpha}, \quad|\partial \Omega|_{m}=\frac{c_{1}}{\alpha c_{0}^{(\gamma+1) / \alpha}} \int_{0}^{\infty} F(r) r^{-2+\frac{\gamma+1}{\alpha}} d r .
$$


Proof. As $r_{i} / l_{i} \rightarrow 0$ when $i \rightarrow \infty$ (because $\beta>\alpha$ ) we may assume that $r_{i}<l_{i} / 6$ for all $i \geqq 1$. If it is not true for some $i \leqq i_{0}$ one can include the boundaries of the balls $B_{i, j}, i \leqq i_{0}$, to the outer boundary $\partial \Omega_{0}$. that

Let $i^{+}(\varepsilon)=\left(\frac{4}{c_{0} \varepsilon}\right)^{1 / \alpha}, i^{-}(\varepsilon)=\left(\frac{1}{4 c_{0} \varepsilon}\right)^{1 / \alpha}$. Since $l_{i} \sim\left(c_{0} i\right)^{-\alpha}$, there is an $\varepsilon_{0}>0$ such

$$
l_{i}<\varepsilon / 3 \quad \text { if } i \geqq i^{+}(\varepsilon), \quad l_{i}>3 \varepsilon \quad \text { if } i \leqq i^{-}(\varepsilon)
$$

if $\varepsilon \leqq \varepsilon_{0}$. From now on we assume that $0<\varepsilon<\varepsilon_{0}$.

From inequality $r_{i}<l_{i} / 6$ and (81) it follows that $\varepsilon$-neighborhoods $\left(B_{i, j}\right)_{\varepsilon}$ of the balls $B_{i, j}$ are located strictly inside of the corresponding cubes $Q_{i, j}$ if $i<i^{-}(\varepsilon)$, and they cover the layers $L_{i}$ if $i>i^{+}(\varepsilon)$. It allows us to get the following estimate for $\mu_{\varepsilon}=\operatorname{mes}\left((\partial \Omega)_{\varepsilon} \cap \Omega\right)$ :

$$
\sum_{i \geqq i^{+}(\varepsilon)} l_{i} k_{i} \leqq \mu_{\varepsilon} \leqq C \varepsilon\left|\partial \Omega_{0}\right|+\sum_{i \leqq i^{-}(\varepsilon)} \frac{4}{3} \pi\left[\left(r_{i}+\varepsilon\right)^{3}-r_{i}^{3}\right] N_{i}^{2} k_{i}+\sum_{i>i^{-}(\varepsilon)} l_{i} k_{i}
$$

From here and the inequality $\left(r_{i}+\varepsilon\right)^{3}-r_{i}^{3} \leqq 6 \varepsilon\left(r_{i}^{2}+\varepsilon^{2}\right)$ it follows that

$$
C_{1} \sum_{i \geqq\left(\frac{4}{c_{0} \varepsilon}\right)^{1 / \alpha}} i^{\gamma-\alpha} \leqq \mu_{\varepsilon} \leqq C \varepsilon\left|\partial \Omega_{0}\right|+C_{2} \varepsilon \sum_{i \leqq\left(\frac{1}{4 c_{0} \varepsilon}\right)^{1 / \alpha}} i^{2 \alpha+\gamma}\left(i^{-2 \beta}+\varepsilon^{2}\right)+C_{3} \sum_{i>\left(\frac{1}{4 c_{0} \varepsilon}\right)^{1 / \alpha}} i^{\gamma-\alpha}
$$

and therefore there exist constants $A_{1}, A_{2}>0$ such that

$$
A_{1} \varepsilon^{1-\frac{\gamma+1}{\alpha}}<\mu_{\varepsilon}<A_{2} \varepsilon^{1-\frac{\gamma+1}{\alpha}} .
$$

One can rewrite these inequalities in the form

$$
A_{1} \varepsilon^{3-m}<\mu_{\varepsilon}<A_{2} \varepsilon^{3-m}, \quad m=2+\frac{\gamma+1}{\alpha},
$$

which shows that the Minkowski dimension $m(\partial \Omega)$ is equal to $2+\frac{\gamma+1}{\alpha}$ if $\partial \Omega$ is Minkowski measurable. To get the measurability we must prove the existence of the limit (Minkowski content) $|\partial \Omega|_{m}:=\lim _{\varepsilon \rightarrow 0} \mu_{\varepsilon} / \varepsilon^{3-m}$.

Now we are going to evaluate $\mu_{\varepsilon}$ more carefully in order to prove the existence of the Minkowski content. We represent $\mu_{\varepsilon}$ in the form of the sum of three terms $\mu_{\varepsilon}^{\prime}+\mu_{\varepsilon}^{\prime \prime}+\mu_{\varepsilon}^{\prime \prime \prime}$, where $\mu_{\varepsilon}^{\prime}$ is the contribution to $\mu_{\varepsilon}$ of the $\varepsilon$-neighborhood of the outer boundary $\partial \Omega_{0}$ and of the balls $B_{i, j}$ with $i<i^{-}(\varepsilon), \mu_{\varepsilon}^{\prime \prime}$ is the contribution to $\mu_{\varepsilon}$ of the $\varepsilon$-neighborhood of the balls $B_{i, j}$ with $i \in\left[i^{-}(\varepsilon), i^{+}(\varepsilon)\right], \mu_{\varepsilon}^{\prime \prime \prime}$ is the contribution to $\mu_{\varepsilon}$ of the $\varepsilon$-neighborhood of the balls $B_{i, j}$ with $i>i^{+}(\varepsilon)$. We will start with the evaluation of $\mu_{\varepsilon}^{\prime \prime}$.

The centers $x_{i, j}$ of balls $B_{i, j}$, inside of each group $\Gamma_{i}$ of layers of the cube $Q$, have a structure of the lattice with the step $l_{i}$. Let's fix $i \in\left[i^{-}(\varepsilon), i^{+}(\varepsilon)\right]$ and consider cubes $Q_{i, j}^{\prime}$ with the vertices at centers $x_{i, j}$ of the balls $B_{i, j}$. These cubes (there are $\left(N_{i}-1\right)^{2}\left(k_{i}-1\right)$ of them) cover completely the group $\Gamma_{i}$ of the layers except for the $l_{i} / 2$-neighborhood of the boundary of the group. The volume of the noncovered part has order $\varepsilon$. If we take all values of $i \in\left[i^{-}(\varepsilon), i^{+}(\varepsilon)\right]$, then the volume of noncovered sets has the order $O\left(\varepsilon\left(i^{+}(\varepsilon)-i^{-}(\varepsilon)\right)\right)=O\left(\varepsilon^{1-1 / \alpha}\right)$. It leads to the following upper estimate for $\mu_{\varepsilon}^{\prime \prime}$ :

$$
\mu_{\varepsilon}^{\prime \prime} \leqq \sum_{i \in\left[i^{-}(\varepsilon), i^{+}(\varepsilon)\right]} F\left(\frac{r_{i}+\varepsilon}{l_{i}}\right) l_{i}^{3}\left(N_{i}-1\right)^{2}\left(k_{i}-1\right)+O\left(\varepsilon^{1-1 / \alpha}\right) .
$$


On the other hand, it is obvious that

$$
\mu_{\varepsilon}^{\prime \prime} \geqq \sum_{i \in\left[i-(\varepsilon), i^{+}(\varepsilon)\right]} F\left(\frac{\varepsilon}{l_{i}}\right) l_{i}^{3}\left(N_{i}-1\right)^{2}\left(k_{i}-1\right) .
$$

From (79) and the Euler-Maclauren formula it follows that

$$
\begin{aligned}
& \quad \sum_{i \in\left[i-(\varepsilon), i^{+}(\varepsilon)\right]} F\left(\frac{\varepsilon}{l_{i}}\right) l_{i}^{3}\left(N_{i}-1\right)^{2}\left(k_{i}-1\right) \sim \sum_{\left(\frac{1}{4 c_{0} \varepsilon}\right)^{1 / \alpha} \leqq i \leqq\left(\frac{4}{c_{0}{ }^{\varepsilon}}\right)^{1 / \alpha}} F\left(\varepsilon c_{0} i^{\alpha}\right) \frac{c_{1}}{c_{0}} i^{\gamma-\alpha} \\
& \sim \frac{c_{1}}{c_{0}} \varepsilon^{\frac{\alpha-\gamma-1}{\alpha}} \int_{\left(1 / 4 c_{0}\right)^{1 / \alpha}}^{\left(4 / c_{0}\right)^{1 / \alpha}} F\left(c_{0} x^{\alpha}\right) x^{\gamma-\alpha} d x \\
& =\frac{c_{1}}{\alpha c_{0}^{(\gamma+1) / \alpha}} \varepsilon^{\frac{\alpha-\gamma-1}{\alpha}} \int_{1 / 4}^{4} F(r) r^{\frac{\gamma+1-2 \alpha}{\alpha}} d r \quad \text { as } \varepsilon \rightarrow 0 .
\end{aligned}
$$

Since $r_{i} \sim c_{2} i^{-\beta}=o(\varepsilon)$ when $i \in\left[i^{-}(\varepsilon), i^{+}(\varepsilon)\right]$, the same estimate is valid for the sum in the right-hand side of (83), and therefore from (83), (84) it follows that

$$
\mu_{\varepsilon}^{\prime \prime} \sim \frac{c_{1}}{\alpha c_{0}^{(\gamma+1) / \alpha}} \varepsilon^{\frac{\alpha-\gamma-1}{\alpha}} \int_{1 / 4}^{4} F(r) r^{-2+\frac{\gamma+1}{\alpha}} d r \quad \text { as } \varepsilon \rightarrow 0
$$

We can repeat arguments used for getting (82) and obtain estimate (82) for $\mu_{\varepsilon}^{\prime}+\mu_{\varepsilon}^{\prime \prime \prime}$ with better constants:

$$
a_{1} \varepsilon^{1-\frac{\gamma+1}{\alpha}}<\mu_{\varepsilon}^{\prime}+\mu_{\varepsilon}^{\prime \prime \prime}<a_{2} \varepsilon^{1-\frac{\gamma+1}{\alpha}} .
$$

It is not difficult to check that if we use constants $1 / n$, $n$ with $n>4$ instead of $1 / 4,4$ in the definition of $i^{-}(\varepsilon), i^{+}(\varepsilon)$, then relation (85) remains valid with limits of integration $1 / n, n$ and $a_{1}, a_{2} \rightarrow 0$ as $n \rightarrow \infty$. It means that

$$
\mu_{\varepsilon} \sim \frac{c_{1}}{\alpha c_{0}^{(\gamma+1) / \alpha}} \varepsilon^{\frac{\alpha-\gamma-1}{\alpha}} \int_{0}^{\infty} F(r) r^{-2+\frac{\gamma+1}{\alpha}} d r \quad \text { as } \varepsilon \rightarrow 0,
$$

and the proof of the theorem is completed.

3.2. $(\alpha, \beta)$-model. It is the same type of domains as in the $(\alpha, \beta, \gamma)$-model but with $\gamma=0$. To be more exact we do not assume the existence of any groups of layers of equal width. In other words $k_{i}=1$ for all $i$. Thus, cube $Q$ is divided into layers $L_{n}$, $n=1,2, \ldots$, of the widths $\Delta_{n}$ with $\Delta_{n} \sim \frac{1}{c_{0} n^{\alpha}}$, the layers are covered by elementary subcubes $Q_{n, j}$, and $\Omega=\Omega_{0} \backslash\left(\cup B_{n, j}\right)$, where $B_{n, j}$ are balls of radii $r_{n}$ centered at the centers of cubes $Q_{n, j}$. As earlier $r_{n} \sim c_{2} n^{-\beta}$, and $\beta>\alpha>1$.

The previous analysis cannot be applied here directly because assumption $\gamma>0$ was used essentially. Instead of assumption $\gamma>0$ we assume now that sequence $\Delta_{n}$ is regular in the sense that $\Delta_{n}-\Delta_{n+1} \sim c n^{-\alpha-1}$ as $n \rightarrow \infty$. In order to formulate an analog of Theorem 17 we need the following notations. Let $P$ be the parallelepiped formed from the unit cube $0 \leqq x, y, z \leqq 1$ by shifting its upper face at the vector $(a, b, 0)$. Let $F(a, b, r)$ be the volume of the part of parallelepiped covered by the 
balls of radii $r$ centered at the vertices of $P$. Let $H(r)$ be the "mean value" of $F(a, b, r)$ with respect to shifts:

$$
H(r)=4 \int_{0}^{1 / 2} \int_{0}^{1 / 2} F(a, b, r) d a d b .
$$

Theorem 18. If $\beta>\alpha>1$ and regularity condition on $\Delta_{n}$ is fulfilled, then boundary of the domain $\Omega=\Omega_{0} \backslash \bigcup_{n, j} B_{n, j} \in \Re^{3}$ is Minkowski measurable with

$$
m(\partial \Omega)=2+\frac{1}{\alpha}, \quad|\partial \Omega|_{m}=\frac{1}{\alpha c_{0}^{1 / \alpha}} \int_{0}^{\infty} H(r) r^{-2+\frac{1}{\alpha}} d r .
$$

The proof is similar to the proof of Theorem 17, and it is based on a special partition of $Q$ by the union of the convex polyhedra with the vertices at centers of the balls $B_{n, j}$. The majority of these polyhedra are "almost parallelepipeds" similar to $P$ (with the upper faces shifted with respect to the lower faces). The shifts are uniformly distributed, and it allows to express $\mu_{\varepsilon}$ through function $H$. Formal analysis is not trivial and slightly bulky. But all technical details can be reconstructed.

\section{Planar Bubble Fractals}

The objective of this section is to prove an analogue of Theorem 7 for the case of the 2-dimensional domain $\Omega$. As it was mentioned in the introduction, the simplest case of 3-d web type domains can be reduced to the planar bubble fractals.

Definition 19. A planar domain $\Omega \subset \Re^{2}$ is called a domain of bubble type if $\Omega=$ $\Omega_{0} \backslash\left(\bigcup_{j=1}^{\infty} B_{j}\right)$, where $\Omega_{0}$ is a bounded domain with the smooth boundary and $\left\{B_{j}\right\}$ is a set of nonintersecting circles $B_{j} \subset \Omega_{0}$ of radii $r_{j}$ with centers at $x_{j} \in \Omega_{0}$.

In this article we additionally assume that $\sum_{j=1}^{\infty} \frac{1}{\left|\ln r_{j}\right|}<\infty$.

We consider the Green function $G_{\lambda}(x, y)$ of the problem

$$
(\Delta-\lambda) G_{\lambda}=\delta(x-y), \quad x, y \in \Omega \subset \Re^{2} ; \quad G_{\lambda}=0 \quad \text { when } x \in \partial \Omega .
$$

Let us mention that the operator $\Delta-\lambda$ in $\Re^{2}$ has a unique fundamental solution $E=E_{\lambda}(x)$ decaying at infinity. This solution has a form

$$
E_{\lambda}(x)=-\frac{1}{2 \pi} K(\sqrt{\lambda} x),
$$

where $K(x)=K_{0}(x)$ is the modified Hankel function: $K(x)=\frac{1}{2} \pi i H_{0}^{(1)}(i x)$.

Function $G_{\lambda}(x, y)$ (as well as $E_{\lambda}(x-y)$ ) has a logarithmic singularity as $x \rightarrow y$. Thus we cannot expect that Weyl's formula will be valid for the integral

$$
\int_{\Omega} G_{\lambda}(x, x) d x .
$$

However in the 2-dimensional case one can avoid the necessity to consider an iteration of the resolvent. We will consider the difference $G_{\lambda}(x, y)-E_{\lambda}(x-y)$. 
Unlike the 3-dimensional case, this difference has a limit as $x \rightarrow y$, and the limit is integrable over $\Omega$. Since we subtracted the fundamental solution from the Green function, Weyl's formula in the case of the 2-dimensional domain $\Omega_{0}$ with the smooth boundary gives the following result:

$$
\int_{\Omega} \lim _{y \rightarrow x}\left[G_{\lambda}(x, y)-E_{\lambda}(x-y)\right] d x=\frac{\operatorname{mes}\left(\partial \Omega_{0}\right)}{4 \sqrt{\lambda}}+o\left(\lambda^{-\frac{1}{2}}\right) .
$$

Now we formulate the main result of this section.

Theorem 20. Let $\Omega$ be a planar domain of bubble type and $\sum \frac{1}{\left|\ln r_{i}\right|}<\infty$. Then

$$
\int_{\Omega} \lim _{y \rightarrow x}\left[G_{\lambda}(x, y)-E_{\lambda}(x-y)\right] d x=\frac{\operatorname{mes} \partial \Omega_{0}+2 \pi \sum r_{i}}{4 \sqrt{\lambda}}+o\left(\frac{1}{\sqrt{\lambda}}\right),
$$

as $\lambda \rightarrow \infty$.

To prove Theorem 20 we need two lemmas. Let us denote by $T_{\lambda}(x, y ; R)$ the Green function of the problem (87) in the exterior of the circle $B(R)$ of the radius $R$ with the center at the origin:

$$
\begin{gathered}
(\Delta-\lambda) T_{\lambda}(x, y ; R)=\delta(x-y), \quad|x|,|y|>R ;\left.\quad T_{\lambda}\right|_{|x|=R}=0 ; \\
T_{\lambda} \rightarrow 0 \quad \text { as }|x| \rightarrow \infty .
\end{gathered}
$$

Let us represent this function in the form

$$
T_{\lambda}(x, y ; R)=E_{\lambda}(x-y)-\widetilde{T}_{\lambda}(x, y ; R) .
$$

Lemma 21. The following estimate is valid for function $\widetilde{T}_{\lambda}$ :

$$
\left|\widetilde{T}_{\lambda}(x, y ; R)\right| \leqq \frac{K(\sqrt{\lambda} x)}{2 \pi K(\sqrt{\lambda} R)} K(\sqrt{\lambda}(|y|-R)) .
$$

Proof. From (90) it follows that

$$
\widetilde{T}_{\lambda}(x, y ; R)=-\frac{1}{2 \pi} K(\sqrt{\lambda}|x-y|) \text { when }|x|=R,|y|>R .
$$

Since function $K(\xi)>0$ and decays monotonically when $\xi>0$, we get from (92) that

$$
\left|\widetilde{T}_{\lambda}(x, y ; R)\right| \leqq \frac{1}{2 \pi} K(\sqrt{\lambda}(|y|-R)) \text { when }|x|=R,|y|>R .
$$

Inequality (91) is a direct consequence of (93) and the maximum principle for operator $\Delta-\lambda$ in $B(R)$. Lemma 21 is proved.

Lemma 22. The following estimates are valid for integrals of $\widetilde{T}_{\lambda}(x, x)$ :

$$
\begin{gathered}
\int_{|x|>R}\left|\widetilde{T}_{\lambda}(x, x ; R)\right| d x \leqq \frac{C}{\lambda|\ln \sqrt{\lambda} R|} \quad \text { if } \sqrt{\lambda} R<\frac{1}{2} ; \\
\int_{|x|>R}\left|\widetilde{T}_{\lambda}(x, x ; R)\right| d x \leqq C\left(\frac{R}{\sqrt{\lambda}}+\frac{\sqrt{R}}{\lambda^{3 / 4}}\right) \quad \text { if } \sqrt{\lambda} R \geqq \frac{1}{2} .
\end{gathered}
$$


Proof. Function $K(\xi)$ has a logarithmic singularity at point $\xi=0$ and it decays at infinity as $\xi^{-1 / 2} e^{-\xi}$. Thus,

$$
\begin{aligned}
& C_{1}|\ln \xi| \leqq K(\xi) \leqq C_{2}|\ln \xi|, \quad 0<\xi<\frac{1}{2}, \\
& C_{1} \frac{1}{\sqrt{\xi}} e^{-\xi} \leqq K(\xi) \leqq C_{2} \frac{1}{\sqrt{\xi}} e^{-\xi}, \quad \xi \geqq \frac{1}{2} .
\end{aligned}
$$

First we consider the case when $\sqrt{\lambda} R<\frac{1}{2}$. From monotonicity of $K(\xi)$ and Lemma 21 it follows that

$$
\left|\widetilde{T}_{\lambda}(x, y ; R)\right| \leqq \frac{K^{2}(\sqrt{\lambda}(|x|-R))}{2 \pi K(\sqrt{\lambda} R)} .
$$

Together with (96) it leads to the estimate

$$
\begin{aligned}
& \int_{R+\frac{1}{2 \sqrt{\lambda}}>|x|>R}\left|\widetilde{T}_{\lambda}(x, x ; R)\right| d x \\
\leqq & C \int_{R}^{R+\frac{1}{2 \sqrt{\lambda}}} \frac{r \ln ^{2} \sqrt{\lambda}(r-R)}{|\ln \sqrt{\lambda} R|} d r=C \int_{0}^{\frac{1}{2 \sqrt{\lambda}}}(r+R) \frac{\ln ^{2} \sqrt{\lambda} r}{|\ln \sqrt{\lambda} R|} d r \\
= & \frac{C}{|\lambda \ln \sqrt{\lambda} R|} \int_{0}^{\frac{1}{2}}(\xi+\sqrt{\lambda} R) \ln ^{2} \xi d \xi \leqq \frac{C_{1}}{\lambda|\ln \sqrt{\lambda} R|}
\end{aligned}
$$

because $\sqrt{\lambda} R<\frac{1}{2}$. Further, from (96)-(98) we get that

$$
\begin{aligned}
& \int_{|x|>R+\frac{1}{2 \sqrt{\lambda}}}\left|\widetilde{T}_{\lambda}(x, x ; R)\right| d x \\
& \leqq C \int_{R+\frac{1}{2 \sqrt{\lambda}}}^{\infty} r \frac{e^{-2 \sqrt{\lambda}(r-R)}}{\sqrt{\lambda}(r-R)|\ln \sqrt{\lambda} R|} d r=C \int_{\frac{1}{2 \sqrt{\lambda}}}^{\infty}(r+R) \frac{e^{-2 \sqrt{\lambda} r}}{\sqrt{\lambda} r|\ln \sqrt{\lambda} R|} d r \\
& =\frac{C}{\lambda|\ln \sqrt{\lambda} R|} \int_{\frac{1}{2}}^{\infty}(\xi+\sqrt{\lambda} R) \frac{e^{-2 \xi}}{\xi} d \xi \leqq \frac{C_{2}}{\lambda|\ln \sqrt{\lambda} R|} .
\end{aligned}
$$

Together with (99) it proves (94).

Now we assume that $\sqrt{\lambda} R>1 / 2$. From (96) and (97) it follows that $K(\xi) \leqq$ $C(1+|\ln \xi|)$ for any $\xi>0$. Together with Lemma 21 it gives the following estimate:

$$
\left|\widetilde{T}_{\lambda}(x, y ; R)\right| \leqq C \frac{K(\sqrt{\lambda}|x|)}{K(\sqrt{\lambda} R)}[1+|\ln (\sqrt{\lambda}(|y|-R))|] .
$$

Thus, if $\sqrt{\lambda} R>1 / 2$ we have

$$
\left|\widetilde{T}_{\lambda}(x, x ; R)\right| \leqq C \sqrt{\frac{R}{|x|}} e^{-\sqrt{\lambda}(|x|-R)}[1+|\ln \sqrt{\lambda}(|x|-R)|], \quad|x|>R .
$$


Hence

$$
\begin{aligned}
\int_{|x|>R}\left|\widetilde{T}_{\lambda}(x, x ; R)\right| d x & \leqq C \sqrt{R} \int_{R}^{\infty} \sqrt{r} e^{-\sqrt{\lambda}(r-R)}[1+|\ln \sqrt{\lambda}(r-R)|] d r \\
& =C \sqrt{R} \int_{0}^{\infty} \sqrt{r+R} e^{-\sqrt{\lambda} r}[1+|\ln \sqrt{\lambda} r|] d r \\
& =\frac{C \sqrt{R}}{\lambda^{3 / 4}} \int_{0}^{\infty} \sqrt{\xi+\sqrt{\lambda}} e^{-\xi}[1+|\ln \xi|] d \xi \\
& \leqq C_{3} \frac{\sqrt{R}}{\lambda^{3 / 4}}+C_{4} \frac{R}{\sqrt{\lambda}},
\end{aligned}
$$

because $\sqrt{\xi+\sqrt{\lambda} R} \leqq \sqrt{\xi}+\sqrt{\sqrt{\lambda} R}$. Lemma 22 is proved.

Proof of Theorem 20. Proof of Theorem 20 is similar to the proof of Theorem 7. The last one is based on Lemmas 12-15. Lemma 22 is substituted for Lemma 12 when the dimension is two.

Lemmas 13,14 have the following 2-dimensional analogues respectively: Let $T_{i}$ be the Green function of the problem (87) for the exterior of the circle $B_{i}$ (i.e. $\left.T_{i}=T_{\lambda}\left(x-x_{i}, y-y_{i} ; r_{i}\right)\right)$ and $\widetilde{T}_{i}=E_{\lambda}-T_{i}$. Then

$$
\begin{gathered}
0>\widetilde{G}_{\lambda}, \widetilde{G}_{\lambda}^{N}, \widetilde{T}_{i} \geqq E \quad \text { where } E=E_{\lambda}(x-y)=-\frac{1}{2 \pi} K(\sqrt{\lambda}|x-y|) ; \\
0 \geqq \widetilde{G}_{\lambda}-\widetilde{G}_{\lambda}^{N} \geqq \sum_{i \geqq N} \widetilde{T}_{i}, \quad x, y \in \Omega .
\end{gathered}
$$

These relations are based on the maximum principle for operator $\Delta-\lambda$ and can be proved in the same way as Lemmas 13, 14.

Instead of Lemma 15 we have now the following estimate. Let $P_{\lambda}$ be the Green function (87) for a planar domain $Q$ with the smooth boundary and $\widetilde{P}_{\lambda}=E_{\lambda}(x-y)$ $-P_{\lambda}(x, y)$. Then, similar to Lemma 15 we have

$$
\left|\widetilde{P}_{\lambda}(x, x)\right| \leqq 4 K(\sqrt{\lambda} \rho), \quad \rho=\rho(x, \partial Q)
$$

For an arbitrary $\varepsilon>0$ we choose $N_{1}=N_{1}(\varepsilon)$ such that

$$
\sum_{i \geqq N_{1}} \frac{C}{\lambda\left|\ln \sqrt{\lambda} r_{i}\right|}+\sum_{i \geqq N_{1}} C\left(\frac{r_{i}}{\sqrt{\lambda}}+\frac{\sqrt{r_{i}}}{\lambda^{3 / 4}}\right) \leqq \frac{\varepsilon}{4 \sqrt{\lambda}},
$$

where $r_{i}$ are the radii of circles $B_{i}$ and $C$ are the constants determined in (94), (95). The existence of $N_{1}(\varepsilon)$ follows from the convergence of the series $\sum \frac{1}{\left|\ln r_{i}\right|}$. From (101), Lemma 22 and (103) we get

$$
\int_{\Omega}\left|\widetilde{G}_{\lambda}(x, x)-\widetilde{G}_{\lambda}^{N}(x, x)\right| d x \leqq \frac{\varepsilon}{4 \sqrt{\lambda}} \quad \text { if } N \geqq N_{1}(\varepsilon) .
$$


After this, we find $N_{2}=N_{2}(\varepsilon)$ such that

$$
\begin{aligned}
\sum_{i \geqq N_{2}} 8 \pi \int_{0}^{r_{i}} K(\sqrt{\lambda} \rho) \rho d \rho & \leqq \sum_{i \geqq N_{2}} 8 \pi r_{i} \int_{0}^{\infty} K(\sqrt{\lambda} \rho) d \rho \\
& \leqq \sum_{i \geqq N_{2}} \frac{8 \pi r_{i}}{\sqrt{\lambda}} \int_{0}^{\infty} K(\rho) d \rho \leqq \frac{\varepsilon}{4 \sqrt{\lambda}}
\end{aligned}
$$

and $N_{3}=N_{3}(\varepsilon)$ such that

$$
\sum_{i \geqq N_{3}} 2 \pi r_{i} \leqq \frac{\varepsilon}{4}
$$

Let $N=\max \left(N_{1}, N_{2}, N_{3}\right)$. Formula (88) implies

$$
\left|\int_{\Omega_{N}} \widetilde{G}_{\lambda}^{N}(x, x) d x-\frac{\operatorname{mes}\left(\partial \Omega_{N}\right)}{4 \sqrt{\lambda}}\right| \leqq \frac{\varepsilon}{4 \sqrt{\lambda}}
$$

if $\lambda \geqq \lambda_{1}(\varepsilon)$ and $\lambda_{1}(\varepsilon)$ is big enough. Together with (106) it leads to the estimate

$$
\left|\int_{\Omega_{N}} \widetilde{G}_{\lambda}^{N}(x, x) d x-\frac{\operatorname{mes}\left(\partial \Omega_{0}+2 \pi \sum r_{i}\right)}{4 \sqrt{\lambda}}\right| \leqq \frac{\varepsilon}{2 \sqrt{\lambda}}, \quad \lambda \geqq \lambda_{1}(\varepsilon) .
$$

From (102) with $Q=\Omega^{N}$ and $P_{\lambda}=G_{\lambda}$, and from (105) we get that

$$
\int_{i \geq N}\left|\widetilde{G}_{\lambda}^{N}(x, x)\right| d x \leqq \frac{\varepsilon}{4 \sqrt{\lambda}} .
$$

Together with (107) and (104) it gives the assertion of Theorem 20. The proof is completed.

\section{References}

[Be] Berry, M.V.: Some geometric aspects of wave motion: Wavefront dislocations, diffraction catastrophes, diffractals. In: "Geometry of the Laplace Operator," Proc. Symp. Pure Math., Vol. 36, Providence, RI: Am. Math. Soc., 1980, pp. 13-38

[BrCa] Brossard, J., Carmona, R.: Can one hear the dimension of a fractal? Commun. Math. Phys. 104, 103-122 (1986)

[FlVa] Fleckinger, J., Vasil'ev, D.: Tambour fractal: Example d'une formule asymptotique à deux termes pour la "foction de comptage." C.R. Acad. Sci. Paris 311, Série 1, 867$872(1990)$

[FlVa2] Fleckinger, J., Vasiliev, D.: An example of a two-term asymptotics for the "counting function" of a fractal drum, Trans. Am. Math. Soc. 337, No 1, 99-117 (1993)

[HuSl] Hua, C., Sleeman, B.D.: Fractal drums and the n-dimensional modified Weyl-Berry conjecture. Comm. Math. Phys. 168, 581-607 (1995)

[JaMoSi] Jaksič, V., Molčanov, S., Simon, B.: Eigenvalue asymptotics of the Neumann Laplacian of Regions and Manifolds with cusps. J. Funct. Anal. 106, No 1, 59-79 (1992)

[Iv1] Ivrii, V.Ja.: Second term of the spectral asymptotic expansion of the Laplace-Beltrami operator on manifolds with boundary. Funct. Anal. Appl. 14, 98-106 (1980)

[Iv2] Ivrii, V.Ja.: "Precise spectral asymptotics for elliptic operators." Lect. Notes in Math. 1100,1984

[Ka] Kac, M.: Can one hear the shape of a drum? Am. Math. Monthly (Slaught Mem. Papers, No.11) 73, 1-23 (1966) 
[La1] Lapidus, M.L.: Fractal drum, inverse spectral problems for elliptic operators and a partial resolution of the Weyl-Berry conjecture. Trans. Am. Math. Soc. 325, 465-529 (1991)

[La2] Lapidus, M.L.: Spectral and fractal geometry: From Weyl-Berry conjecture for the vibrations of fractal drums to the Riemann zeta-function. In: "Differential Equations and Mathematical Physics," Proc. UAB Intern. Conf. (Birmingham, 1990), New York: Academic Press, 1992, pp. 151-182

[LaF1] Lapidus, M.L., Fleckinger-Pellé, J.: Tambour fractal: vers une resolution de la conjecture de Weyl-Berry pour les valeurs propresdu laplacien, C.R. Acad. Sci. Paris Sér. 1 Math. 306, 171-175 (1988)

[LaPo] Lapidus, M.L., Pomerance, C.: Countrexamples to the modified Weyl-Berry conjecture on fractal drums. Preprint

[LeVa] Levitin, M., Vassiliev, D.: Spectral asymptotics, renewal theorem, and the Berry conjecture for a class of fractals, Proc. London Math. Soc. (3) 72, 188-214 (1996)

[Ma] Mandelbrot, B.B.: "The Fractal Geometry of Nature." Rev. and enl. ed., New York: W.H. Freeman, 1983

[Me] Melrose, R.B.: Weyl's conjecture for manifolds with concave boundary. In: "Geometry of the Laplace Operator," Proc. Symp. Pure Math. 36 (1980), Providence, RI: Am. Math. Soc., pp. 254-274

[McSi] McKean, H.P., Singer, I.M.: Curvature and the eigenvalues of the Laplacian. J. Differ. Geom. 1, 43-69 (1967)

[Mo] Molchanov, S.: Diffusion processes and Riemann geometry. Russ. Math. Surv. 30, No. 1, 3-60 (1975)

[Se] Seeley, R.T.: A sharp asymptotic remainder estimate for the eigenvalues of the Laplacian in a domain of $\Re^{3}$. Adv. in Math. 29, 244-269 (1978)

[Si] Simon, B.: The Newmann Laplacian of a jelly roll. Proc. of the AMS 114, No. 3, 783-785 (1992)

Communicated by B. Simon 
